Single Scan Parameterization of Space-Variant Point Spread Functions in Image Space via a Printed Array: Impact for two PET/CT Scanners

Kotasidis, FA and Matthews, JC and Angelis, $\mathrm{Gl}$ and Noonan, PJ and Jackson, A and Price, $P$ and Lionheart, WR and Reader, AJ 2011 MIMS EPrint: 2015.41

Manchester Institute for Mathematical Sciences

School of Mathematics

The University of Manchester

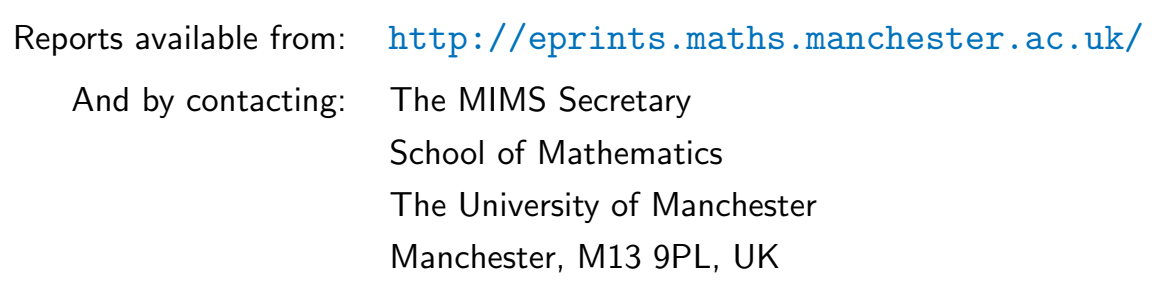




\title{
Single Scan Parameterization of Space-Variant Point Spread Functions in Image Space via a Printed Array: Impact for two PET/C T Scanners
}

\author{
F A Kotasidis ${ }^{1}$, J C Matthews ${ }^{1}$, G I Angelis ${ }^{1}$, P J Noonan ${ }^{1}$, A Jackson ${ }^{1}$, P Price ${ }^{3}$, W R Lionheart ${ }^{2}$, \\ A J Reader ${ }^{4}$ \\ ${ }^{1}$ Imaging, Genomics and Proteomics, Wolfson molecular imaging centre, MAHSC, University of Manchester, Manchester, UK \\ ${ }^{2}$ School of Mathematics, Alan Turing Building, University of Manchester, Manchester, UK \\ ${ }^{3}$ Academic Department of Radiation Oncology, University of Manchester, Manchester, UK \\ ${ }^{4}$ Brain Imaging Centre, Montreal Neurological Institute, McGill University, Montreal, QC, Canada \\ E-mail fotis.kotasidis@mmic.man.ac.uk
}

\begin{abstract}
Incorporation of a resolution model during statistical image reconstruction often produces images of improved resolution and signal-to-noise ratio (SNR). A novel and practical methodology to rapidly and accurately determine the overall emission and detection blurring component of the system matrix using a printed point source array within a custom-made Perspex phantom is presented. The array was scanned at different positions and orientations within the field of view (FOV) to examine the feasibility of extrapolating the measured point source blurring to other locations in the FOV and the robustness of measurements from a single point source array scan. We measured the spatially-variant image based blurring on two PET/CT scanners, the B-Hi-Rez and the TruePoint TrueV. These measured spatiallyvariant kernels and the spatially-invariant kernel at the FOV centre were then incorporated within an ordinary Poisson ordered subsets expectation maximization (OP-OSEM) algorithm and compared to the manufacturer's implementation using projection space resolution modelling. Comparisons were based on a point source array, the NEMA IEC image quality phantom, the Cologne resolution phantom and 2 clinical studies (carbon-11 labelled anti-sense oligonucleotide $\left[{ }^{11} \mathrm{C}\right]$-ASO and fluorine-18 labelled fluoro-L-thymidine $\left[{ }^{18} \mathrm{~F}\right]$-FLT). Robust and accurate measurements of spatially-variant image blurring were successfully obtained from a single scan. Spatially-variant resolution modelling resulted in notable resolution improvements away from the centre of the FOV. Comparison between spatiallyvariant image-space methods and the projection-space approach (the first such report, using a range of studies for two distinct PET/CT systems) demonstrated very similar performance with our image-based implementation producing slightly better contrast recovery (CR) for the same level of image roughness (IR). These results demonstrate that image-based resolution modelling within reconstruction is a valid alternative to projection based modelling, and that, when using the proposed practical methodology, the necessary resolution measurements can be obtained from a single scan. This approach avoids the relatively time-consuming and involved procedures previously proposed in the literature.
\end{abstract}




\section{Introduction}

In the last few years tremendous effort has been put into developing image reconstruction algorithms for positron emission tomography (PET). Statistical methods can often provide images of better SNR and resolution compared to conventional analytic algorithms by accounting for differences in measurement precision and allowing extensive modelling of the emission and detection processes occurring during data acquisition. This can be realized through the use of a system matrix but in common practice an over-simplified forward and back-projector is used or a line integral model which approximates only the geometric detection probabilities (Joseph 1982, Siddon 1985). This model, although computationally simple, fails to take into account more complex physical phenomena taking place during data acquisition. In reality emission effects like positron range and photon non-colinearity as well as detection effects like multiple photon interactions within the crystal block, light transport within the crystal array, errors in the crystal identification matrix and errors between the effective and the physical crystal location (Qi et al 1998, Tomic et al 2005), will result in a blurred measurement of the sinogram and subsequently the image.

To correct for these emission/detection blurring effects and accurately model the relationship between image and projection space these effects can be taken into account during the reconstruction process either in projection space or image space.

The measurement of image space point spread function (PSF) kernels, and their use in resolution modelling, has the potential advantage of being able to capture and correct for both emission and detection resolution degrading effects. Image based resolution modelling for PET was first proposed by Reader et al $(2001,2002,2003)$ who used a simple Gaussian PSF model to demonstrate enhanced resolution and noise reduction on clinical images while Sureau et a/ (2008), using a similar model on the HRRT, assessed the impact of such a model on phantom studies and kinetic parameters. Although spatially invariant kernels provide improvements in SNR and resolution, for most scanners which lack depth-of-interaction capabilities use of a spatially variant kernel may be more appropriate. Rahmim et a/ (2003) used a transaxially variant and anisotropic non-Gaussian model over stationary and isotropic kernels while in a much later study (Rahmim et al 2008a) proposed an image space technique that incorporates the spatially-variant, medium-dependent nature of positron range. Recently Rapisarda $e t$ al (2010) used measured spatially variant kernels within an image based resolution modelling reconstruction on the GE Discovery STE while Cloquet et a/ (2010) explored non-Gaussian variant and anisotropic models within a list-mode reconstruction algorithm on the Philips Gemini.

On the other hand, projection space modelling attempts to model both emission and detection resolution degrading effects in sinogram space. A number of studies can be found in the literature, some of which predate the image-space methods, (Mumcuoglu et al 1996, Qi et al 1998, Panin et al 2006, 2007, De Bernardi et a/2007, Wiant et a/2009, Tohme et a/2009, Tong et a/2010, Alessio et al 2010) with the differences between them lying in the dimensionality of the PSF model and the methods used to derive the kernels.

An excellent theoretical analysis of the two resolution modelling approaches can be found in Cloquet et a/ (2010). As noted in this work, modelling of detection blurring effects within image based RM is an approximation. One attractive property of the image based method though is the fact that a fully characterized PSF is a 6-dimensional function (i.e. a unique 3D kernel response which is a function of 3D position in the object), while a fully characterized projection based blurring occupies a 7dimensional space (i.e. a unique 4D kernel response which is a function of 3D position in the object). Furthermore as most of the clinical scanners have list-mode acquisition capability, implementing a listmode resolution modelling reconstruction is only possible if image based methods are used. Nevertheless it remains to be demonstrated whether the image based approach is a valid alternative to the projection based approach.

Although image based and projection based methods are different in terms of their implementation within the image reconstruction, both require estimation of the PSF model. This is done either by analytic derivation (Rahmim et al 2008b,Moehrs et al 2008), Monte Carlo simulations (Alessio et al 2006, Ortuno et a/2006) or by measuring the scanner response to a point source at different locations in the FOV (Panin et a/2006, De Bernardi et a/2007, Wiant et a/2009, Alessio et a/2010).

Deriving the PSF from real point source measurements constitutes the most accurate but also the most challenging approach. Conventionally this method requires a point source positioned and scanned at different but known locations in the FOV. In addition, for projection space RM, in order to derive the angular blurring component in projection space the point source needs to be collimated to avoid crosstalk from adjacent LORs. For that reason typically measurements with an un-collimated point source are used, assuming no blurring in the angular direction. This also enables the use of ordered subsets type reconstruction algorithms since there is no blurring across LORs within different subsets. 
Using a point source, at the location of each image voxel, the blurring kernels can in theory be derived. However in practice this involves a huge number of measurements and restricts the reconstructed image grid to be the same as the one used for the PSF measurement. For that reason interpolation and extrapolation of a limited number of measurements is typically performed, using a parameterized model of the blurring. Using a point source to measure the data blurring is time consuming requiring complex and expensive equipment. Usually sophisticated robots are employed to accurately position and move a single point source throughout the FOV. Panin et al (2006) was the first to use a 3-D positioning robot with a single ${ }^{68} \mathrm{Ge}$ source at 1599 positions in his seminal work while Wiant et al (2009) using a similar design, scanned a single ${ }^{68} \mathrm{Ge}$ source at 6336 points. Tohme et a/ (2009) used a robot and a single ${ }^{22} \mathrm{Na}$ source to sample the PSF at 3064 transaxial positions but restricted the measurements in a single axial plane. Finally Cloquet et a/ (2010) used a single ${ }^{22} \mathrm{Na}$ source and a grid to guide the source at the measured positions. Limited measurements of a ${ }^{22} \mathrm{Na}$ point source were also used by Alessio et al (2010) and Rapisarda et al (2010).

All these devices can only position one point source at a time and with the need for high count statistics, there is a compromise between the number of sampled points and the acquisition duration. The method of producing radioactive point sources using a standard inkjet printer has become increasingly popular. Printed point sources have been previously used in SPECT (Van Staden et al 2007) to produce quality assurance phantoms and also in PET (Sossi et al 2003) for resolution measurements. These studies focused on the feasibility of producing such point sources, demonstrating the simplicity of the method. One complication of positron emitting isotopes though is the need for a medium for positron annihilation, without dramatically affecting counting statistics through attenuation.

In this paper, we propose and assess the use of a printed array of $\left[{ }^{18} \mathrm{~F}\right]$ point sources and a custom made Perspex phantom to rapidly derive the spatially-variant image based blurring component of the system matrix. The impact of such an approach on image quality was then assessed for the Biograph 6 B-Hi-Rez PET/CT and the Biograph 6 TruePoint TrueV PET/CT cameras. Specifically we compared three different fully 3-D ordinary Poisson OSEM (OP-OSEM) resolution modelling algorithms: 1) an in house implementation (OP-OSEM) using the measured image based spatially variant PSF (varIMPSF OP-OSEM); 2) an in house OP-OSEM implementation using the spatially invariant measured image based PSF at the centre of the field of view (invIMPSF OP-OSEM); 3 ) the commercial PSF reconstruction implementation using projection space modelling (varPRPSF OP-OSEM). As a reference we also used an in-house implementation of the standard reconstruction without any resolution modelling (noPSF OP-OSEM). The assessment was carried out using a range of phantom and clinical datasets: point source data; the image quality NEMA phantom data; Cologne resolution phantom data, and clinical data from a $\left[{ }^{11} \mathrm{C}\right]$-ASO and an $\left[{ }^{18} \mathrm{~F}\right]-\mathrm{FLT}$ scan. This constitutes the first direct comparison between image based and projection based resolution modelling reconstructions.

\section{Materials and methods}

\subsection{The B-Hi-Rez scanner}

The Biograph 6 Barrel-Hi-Rez PET/CT (Siemens Molecular Imaging Inc., TN, USA) is a whole body scanner capable of list-mode acquisition (Brambilla et a/2005). The scanner contains three block rings, with each ring consisting of 48 blocks resulting in a $162 \mathrm{~mm}$ and a $585 \mathrm{~mm}$ FOV axially and transaxially respectively. Unlike many PET cameras, the outer ring blocks are positioned at a 7.5 degrees angle to the central ring (figure 1), giving the scanner a spherical geometry. As a result the transaxial FOV is a function of the axial distance from the centre. The data are acquired in span 11 with a maximum ring difference (rd) of 27 and histogrammed into 313 sinograms in 3-D mode, consisting of 336 radial elements and 336 angular views after azimuthal interleaving. Before image reconstruction an 'arc correction' (the rebinning of events into parallel projections of equal width) is performed. Unlike most cylindrical geometry scanners, the spherical geometry of this scanner results in dependency of this rebinning on the axial in addition to the radial element position of the recorded events.

\subsection{The TruePoint TrueV scanner}

The Biograph 6 TruePoint TrueV PET/CT (Siemens Molecular Imaging Inc., TN, USA) is the next generation scanner after the Hi-Rez (Jakoby et al 2006, 2009) and features 2 important additions compared to its predecessor. In order to increase the sensitivity, the scanner has an additional block ring which extends its axial FOV to $21.6 \mathrm{~cm}$ (figure 1). This results in 559 3-D sinograms ( $\operatorname{span}=11$, $\mathrm{rd}=38$ ). Secondly the spherical design of the scanner has been replaced with a more conventional cylindrical one, eliminating the need for axially-dependent arc correction. 

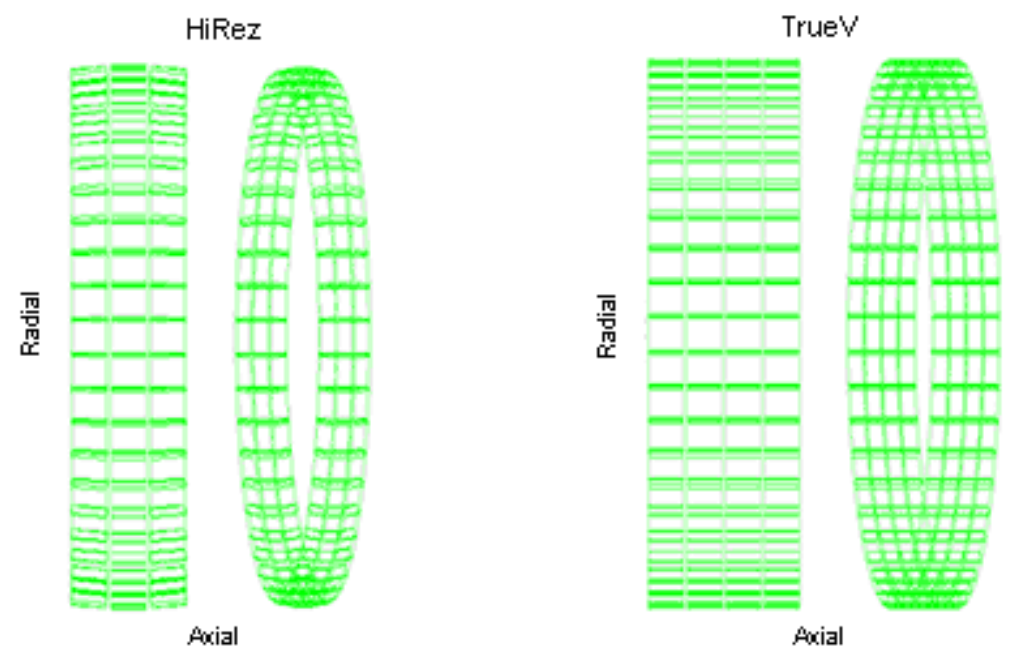

Figure 1. The Biograph 6 B-Hi-Rez (left) and the TruePoint TrueV PET/CT (right) geometric design and block rings.

\subsection{Point source production and optimization}

An HP 5440 printer was used in order to print radioactive point sources at predefined positions on a sheet of A4 paper. Standard black ink was mixed with small quantities of $\left[{ }^{18} \mathrm{~F}\right]$ saline solution $(0.1-0.2$ $\mathrm{ml}$ ) which was injected into an ink cartridge. Prior to injection the ink cartridge was modified by removing the sponge and the membrane which controls the ink delivery inside the cartridge in order to apply ink directly to the reservoir for maximum efficiency. In order to optimally space the sources and choose the best source dimensions, the Hi-Rez scanner was used to scan point sources with various diameters and distances between sources, at various locations in the FOV. This point source spacing optimization has a direct effect on the number of point sources per array. The raw point source data were reconstructed using an OP-OSEM algorithm with fine image voxel sampling (10 iterations, 21 subsets, $0.66 \mathrm{~mm} \times 0.66 \mathrm{~mm} \times 2 \mathrm{~mm}$ voxels) and the resulting images were qualitatively analyzed using line profiles along the radial and axial directions to determine the degree of overlapping of adjacent PSF profiles. Maximization of the activity per point source minimizes acquisition time or maximizes the counting statistics and hence is desirable. Increasing the size of the round dot sources and reprinting of the sources using the same paper were investigated as approaches in order to boost the activity per point source. Larger sources will decrease the accuracy of the PSF measurements, with such degradation assessed qualitatively. Reprinting the point source multiple times will potentially degrade the point source resolution if reprinting does not occur in exactly the same location. The latter was checked through examination of microscope-enhanced images. As a compromise between resolution and activity, a source diameter of $2 \mathrm{~mm}$ was chosen matching the dimensions of the reconstructed voxels typically used. Based on this optimization, the dimensions of the sources and their spacing were used to design the array in Microsoft publisher.

\subsection{Positioning and aligning the point source array}

To position the point sources within the FOV and allow them to move in all 3 dimensions a phantom was designed (figure 2). The phantom was made out of an aluminium bar, located outside the FOV, in which holes were drilled every $2 \mathrm{~cm}$. Attached to the bar were 2 sheets, $3 \mathrm{~mm}$ width each, made out of a tissue equivalent material (Perspex). The use of a transparent material made positioning and alignment of an A4 sheet of paper easier. The width of $3 \mathrm{~mm}$ was chosen as a compromise between positron annihilation and the minimization of attenuation. To move the phantom within the FOV the aluminium bar was attached to the QC phantom holder that was supplied with the scanner and which was subsequently attached to the scanner's bed. The phantom holder as well as the holes in the metal bar allowed movement in the radial and tangential directions while the movement of the bed through the scanner's patient handling system (PHS) was used to cover the axial direction.

The point source alignment was done in 2 steps. First the scanner's laser system was used to align the outermost rows and columns of the array of point sources to the axial and the radial lasers (Figure 2),which correspond to the central axis and the edge of the CT FOV. With the sources positioned, a quick CT scout scan was taken to position the phantom axially. Following a short PET emission scan, the within plane segment zero sinograms were examined to observe the axial deviation of a single radial line of point sources and if necessary correct the array position. When the point sources were perfectly 
aligned with respect to the radial scanner axis they should appear within the same projection plane. Note that only a single alignment is required and that it is quicker and easier than previous methods requiring the alignment of a moving robotically positioned point source (Panin et a/ 2006). Following alignment using the QC phantom holder, the positioning holes within the aluminium bar and the couch, the array could be moved to the desired location.
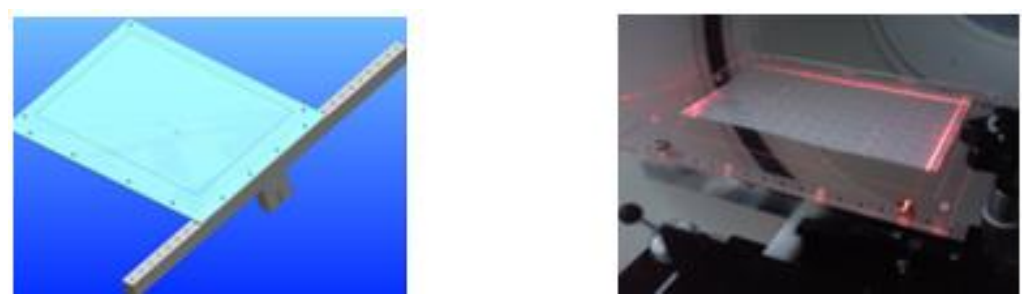

Figure 2. A Perspex phantom was designed (left) to hold the array and provide material for the positron to annihilate. An aluminum bar was used to attach the array to the QC phantom holder. The array after being aligned with lasers was scanned at different positions (right). The axial movement was done by changing the PET/CT FOV with respect to the topogram FOV as the array had always a fixed position with respect to the topogram. In the radial direction, movement was provided by moving the Perspex sheets to different holes (discrete movement- $2 \mathrm{~cm}$ hole-to hole spacing) or by manually shifting the phantom holder radially for finer sampling (continuous movement with sub-millimetre accuracy).

\subsection{Scanning and reconstruction protocol}

2.5.1. Hi-Rez scanner. On the Hi-Rez a rectangular grid of 8 (axially) $\times 15$ (radially) point sources, evenly spaced at $20 \mathrm{~mm}$ was scanned 120 times in order to measure the PSF with a fine spatial sampling over a FOV segment assuming radial and rotational PSF symmetries. In the radial direction the array was scanned at 4 radial positions with a $5 \mathrm{~mm}$ step size moving from the centre towards the edge of the FOV. In the axial direction the array sampled 10 axial positions with a $2 \mathrm{~mm}$ step size. This axial sampling was chosen to coincide with the plane width. In the vertical (approximately tangential) direction the sources were scanned at 3 positions $5 \mathrm{~mm}$ apart. As a result of these scans the PSF is measured for a grid of 60 (radial) $\times 80$ (axial) $\times 3$ (tangential) points $(14400$ in total $)$, spaced $5 \mathrm{~mm}$ radially and tangentially and $2 \mathrm{~mm}$ axially (Figure 3 ). Due to the short 2 hour half-life, it was not possible to perform all 120 scans using the same printed sheet. In practice 6 scanning sessions were performed, each of 4 hours duration (1 array/scanning session). To account for decay within each scanning session and ensure comparable statistics, progressively longer acquisition periods were used (5-20 min).

2.5.2. TrueV TruePoint scanner. On the TruePoint TrueV PET/CT we used a different sampling scheme in order to assess whether the spatially dependent characteristics of the PSF could be measured using a single scan, and to assess the reproducibility using different scans and positioning. The array was made out of a rectangular grid of 9 (axially) $\times 14$ (radially) point sources, with a $20 \mathrm{~mm}$ spacing both in the axial and radial directions. For evaluation and assessment purposes it was scanned at 3 different positions.
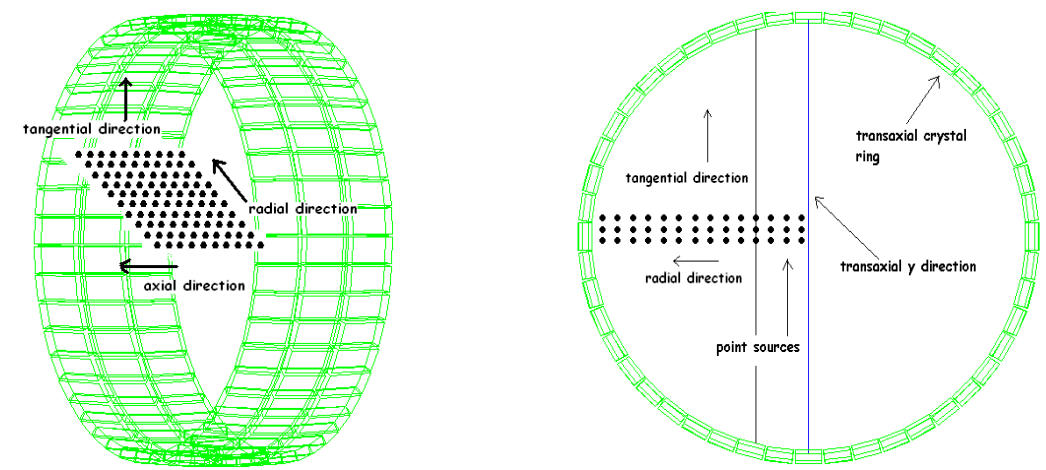

Figure 3. Sampling scheme for the Hi-Rez. The axial extent of the array covered the entire axial FOV and half the transaxial FOV.

In the first scan which we consider to be the reference scan), the axial centre of the array (middle radial line of point sources) was positioned in the centre of the axial FOV $(Z=0 \mathrm{~cm})$ covering almost the entire axial FOV $(Z=-8 \mathrm{~cm} \ldots+8 \mathrm{~cm})$. Transaxially the edge of the array was positioned $1 \mathrm{~cm}$ from the centre of the transaxial FOV $(r=1 \mathrm{~cm} . .27 \mathrm{~cm})$ having a zero azimuthal angle $\left(\varphi=0^{\circ}\right)$ from the 
horizontal scanner axis. To examine whether the axial sampling using a single array is sufficient, we acquired a second scan at the same radial and azimuthal position but shifting the array in the axial direction by $1 \mathrm{~cm}$. Finally in order to determine if the PSF model is rotationally symmetric, the array was scanned with the same axial and radial position of the reference scan but at a different azimuthal angle $\left(\varphi=37^{\circ}\right)$.

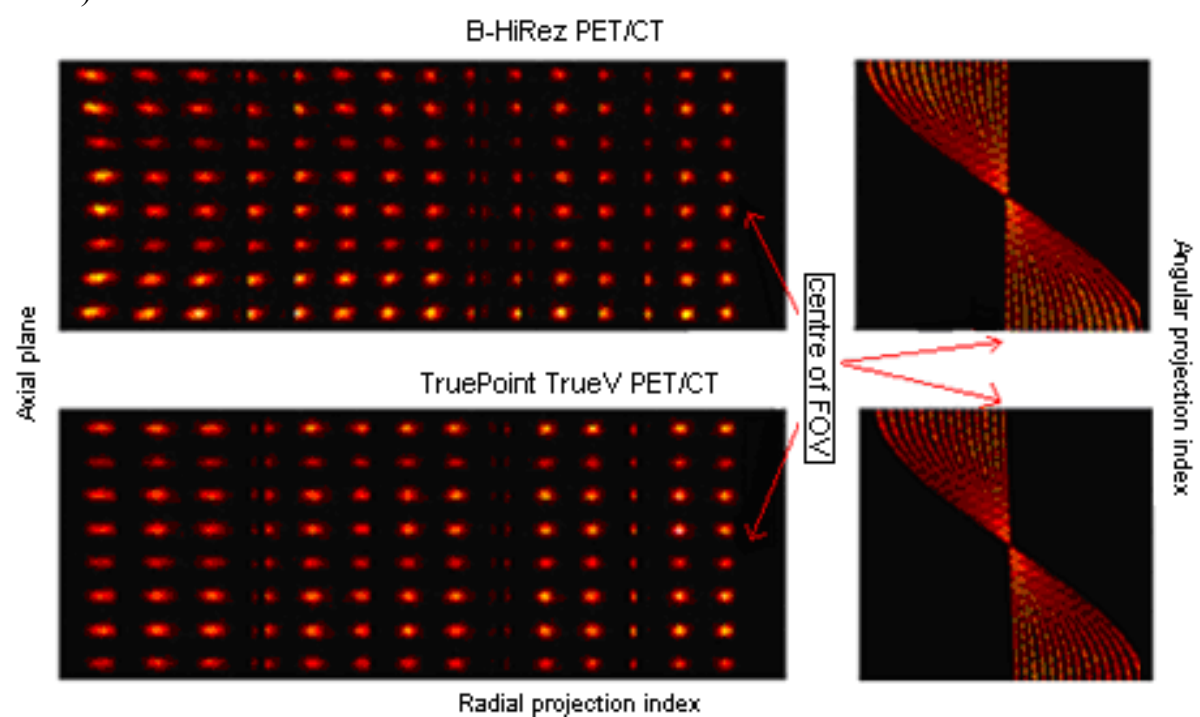

Figure 4. Horizontal (left) and transaxial (right) sections through the 3-D sinogram space. Moving away from the centre of the FOV the radial elongation is obvious. The spherical design of the Hi-Rez compared to the cylindrical geometry of the TrueV elongates the PSFs also in the axial direction with a correlation between the radial and axial blurring components. The gaps between the crystals are seen as black strips along the axial direction especially when an LOR corresponding to a gap passes through a point source.

2.5.3. Reconstruction of point source data for PSF determination. PET data were collected in 32-bit list mode for both scanners and organized into prompt and random events using an in-house list-mode histogrammer, which had previously been cross validated against sinograms produced using the manufacturer's software. Standard Hi-Rez and TrueV software was used for calculating the correction sinograms prior to reconstruction. Prompt data were corrected for geometric effects (axial and radial arc correction for the Hi-Rez and only radial arc correction for the TrueV) while variance reduction was used to smooth the randoms sinogram (delayed coincidence window method). Scatter calculation is based on a 2-D implementation of the single scatter simulation method (Watson et a/ 2004) and then expanded to 3-D. Normalization is indirect component based (geometric effects, crystal interference, crystal efficiencies, axial effects, paralyzing and non-paralyzing ring dead-time parameters) with the dead-time correction being included in the normalization sinogram after taking into account the average singles rate at the detector block level. Finally the standard scanner software uses the CT data to generate the 2-D attenuation correction sinogram. The 3-D attenuation sinogram is then calculated by inverse Fourrier rebinning the 2-D sinogram. Images were reconstructed using an in house 3-D OP-OSEM reconstruction algorithm (10 iterations and 21 subsets), accounting for scatter and randoms. Normalization and attenuation effects were included within a factorized system matrix. Our 3D OPOSEM algorithm made use of a pre-calculated geometric system matrix, based on a 3-D implementation of the Siddon algorithm (Siddon et al 1985). For all the reconstructions a very fine pixel grid was used $(1008$ pixels $\times 1008$ pixels $\times 81$ pixels $)$ to ensure adequate PSF sampling having image voxel dimensions of $0.66 \mathrm{~mm} \times 0.66 \mathrm{~mm} \times 2 \mathrm{~mm}$.

\subsection{PSF parameterization and space variant image-based resolution modelling implementation}

As mentioned in the introduction the PSF data can be used directly if sampled for every voxel in the FOV. Since in practice one can only sample the PSF at a limited number of positions, a PSF model was used in order to fit a number of parameters to the measured data. Each reconstructed PSF was modelled in image space as a mixture of two 3-D Gaussian distributions (with radial, axial and tangential components). The mean for the two Gaussian functions was left unconstrained in order to also take into account the asymmetry in the PSF distributions as a consequence of the parallax error. The use of two Gaussian distributions, potentially with quite different kernel widths enables non-Gaussian distributions to be modelled. Specifically, each PSF was modelled as: 


$$
\begin{gathered}
P S F\left(\mathrm{x}^{\prime}, \mathrm{x}\right)=\sum_{i=1}^{2} w_{i} G_{i}\left(\mathrm{x}^{\prime}, \boldsymbol{\mu}(\mathrm{x}), \boldsymbol{\Sigma}(\mathrm{x})\right), \sum_{i=1}^{2} w_{i}=1 \quad(1) \\
G_{i}\left(\mathrm{x}^{\prime}, \boldsymbol{\mu}(\mathrm{x}), \boldsymbol{\Sigma}(\mathrm{x})\right)=\frac{1}{(2 \pi)^{3 / 2}|\boldsymbol{\Sigma}(\mathrm{x})|^{1 / 2}} \exp \left(-\frac{1}{2}\left(\mathrm{x}^{\prime}-\boldsymbol{\mu}(\mathrm{x})\right)^{T} \boldsymbol{\Sigma}(\mathrm{x})^{-1}\left(\mathrm{x}^{\prime}-\boldsymbol{\mu}(\mathrm{x})\right)\right) \\
\boldsymbol{\mu}=\left(\begin{array}{l}
\mu_{\gamma}(\mathrm{x}) \\
\mu_{\mathrm{t}}(\mathrm{x}) \\
\mu_{\zeta}(\mathrm{x})
\end{array}\right) ; \boldsymbol{\Sigma}=\left(\begin{array}{ccc}
\sigma_{\gamma}^{2}(\mathrm{x}) & 0 & 0 \\
0 & \sigma_{\mathrm{t}}^{2}(\mathrm{x}) & 0 \\
0 & 0 & \sigma_{\zeta}^{2}(\mathrm{x})
\end{array}\right)
\end{gathered}
$$

where $\mathrm{X}$ and $\mathrm{X}^{\prime}$ are position vectors relating to the image space and PSF kernel respectively, $G_{i}$ is the function describing a 3 dimensional Gaussian distribution and $w_{j}$ is a mixing term. The matrices $\boldsymbol{\mu}$ and $\boldsymbol{\Sigma}$ hold the first and second order moments of the PDF (mean and covariance matrix) for the radial, axial and tangential responses. $\boldsymbol{\Sigma}^{-1}$ is the inverse of the matrix $\boldsymbol{\Sigma}$ and superscript $T$ denotes the transpose. The constants in front of the exponential are normalization constants, such that the integral of equation (2) is equal to 1 . The covariance matrix was constrained to non-zero diagonal elements based on the data and to minimize the fitted parameters. A total of 13 parameters (6 parameters for each of the two Gaussian distributions $\left(\mu_{\gamma}, \mu_{t}, \mu_{\zeta}, \sigma_{\gamma}^{2}, \sigma_{t}^{2}, \sigma_{\zeta}^{2}\right)$ and one mixing coefficient $\left.\left(w_{i}\right)\right)$ were estimated for each measured PSF. The Expectation Maximization (EM) algorithm was used to construct an object of the gmdistribution class in Matlab (The Mathworks, R2009a) containing maximum likelihood estimates of the parameters using the above Gaussian mixture model.

The derived model parameters could then be fitted to $2^{\text {nd }}$ order polynomial functions dependent on the radial $(r)$ and axial (z) position of the point source. This enables determination of the model parameters for every position in the FOV. Based on the data analysis from the 2 scanners though, only a very small axial dependency of the axial blurring was seen, with the radial and tangential blurring being practically axially independent. For that reason and to minimize the storage requirements of the overall PSF each parameter was radially dependant, but invariant to axial and rotational transformations.

Each image voxel within an axial plane was then assigned with a set of model parameters based on its radial distance and the PSF's where pre-calculated and stored in a $\mathrm{K}^{2} \times \Theta^{3}$ matrix, where $\mathrm{K}$ is the number of pixel in the plane and $\Theta^{3}$ is the size of the 3-dimensional kernel. Figure 5 shows fitted PSFs with a nearly symmetric distribution at the centre and a radially skewed distribution at the edge of the FOV
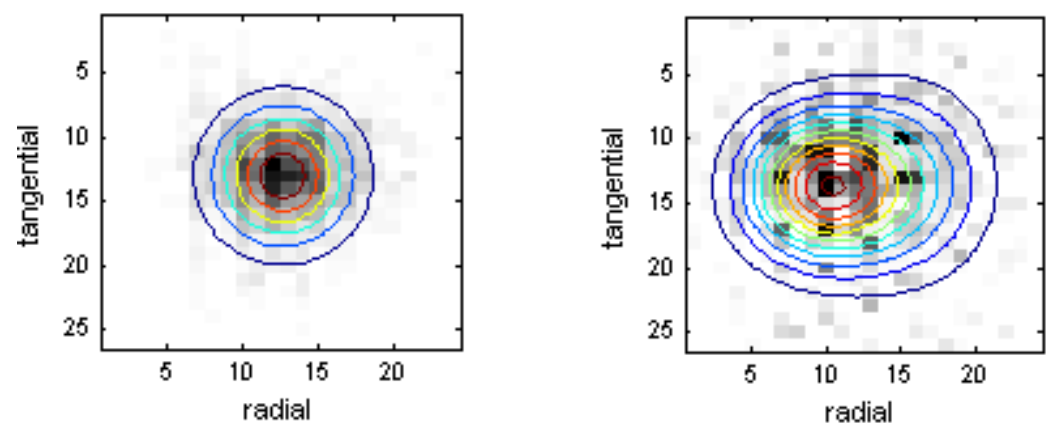

Figure 5. 2-D sections through the 3-D fitted PSFs at $2 \mathrm{~cm}$ (left) and $22 \mathrm{~cm}$ (right) from the centre of the FOV. Close to the centre of the FOV the PSF is almost symmetric while at the edge of the FOV the radial elongation results in an asymmetric distribution.

To implement a spatially-variant image based resolution modelling algorithm we used an ordinary Poisson ordered subsets expectation maximization (OP-OSEM) algorithm and applied the pre-calculated spatially-variant blurring matrix using linear blurring operations during the forward-projection and backprojection steps (equation 4).

$$
\lambda_{l}^{\kappa+1}=\frac{\lambda_{l}^{\kappa}}{\sum_{k} \vartheta_{k, i} s_{k}} \sum_{k} \vartheta_{k, i} \sum_{j} p_{j k} \frac{m_{j}}{\sum_{k} p_{j k} \sum_{i^{\prime}} \vartheta_{k, i} \lambda_{j}^{\kappa}+\eta_{j}}, \sigma_{k}=\sum_{j} p_{j k} n_{j} a_{j}, \eta_{j}=\frac{r_{j}}{a_{j} n_{j}}+\frac{\boldsymbol{s}_{j}}{a_{j}}
$$

where $\sigma_{k}$ is the sensitivity image, $\lambda_{j}$ is the mean number of photon emissions occurring in the th pixel, $\mathrm{m}_{\mathrm{j}}$ is the measured events in the $j$ th sinogram bin, $p_{j k}$ is the geometric probability matrix of events being detected at $j$ th sinogram bin given emitted from $k$ th image pixel (and determined using Siddon's 
algorithm), $a_{j}$ is the probability of an event not being attenuated, $n_{j}$ is the photon detection probability in the detectors, $r_{j}$ and $s_{j}$ are the estimated mean random and scatter events being detected at $f$ th sinogram bin and $\vartheta_{k, i}$ is the PSF kernel representing the blurring of an event emitted from the th voxel to the $k$ th voxel. During the back-projection process the transpose of the blurring kernel is used for the blurring operation. In addition to the spatially-variant PSF reconstruction, the symmetric kernel corresponding to the one obtained at the centre of the FOV was used for all image voxels to produce a spatially-invariant PSF reconstruction.

\subsection{Evaluation of spatially variant and invariant PSF reconstructions}

We evaluated the accuracy and utility of the proposed methodology to determine and correct for image blurring by comparing three in house implementations of OP-OSEM (no resolution modelling (noPSF OP-OSEM); image based space invariant RM (invIMPSF OP-OSEM) ; image based space variant RM (varIMPSF OP-OSEM)) with the manufacturer's projection based reconstruction (varPRPSF OP-OSEM). As the varPRPSF is not yet commercially available on the Hi-Rez, we were only able to compare the image based and projection based resolution modelling reconstruction implementations on the TrueV datasets. For all the datasets, the same pre-processing methods were used as in the reconstruction of the PSF data (section 2.5.3). On the Hi-Rez and TrueV for all resolution recovery reconstructions we used 8 iterations and 21 subsets to match the maximum number of iterations in the manufacturer's implementations. For the non-PSF reconstructions we used 5 iterations and 21 subsets to match the statistical background noise in the PSF reconstructions (Sureau et a/2008). For the point source data we also used 15 iterations and 21 subsets. On the NEMA phantom we reconstructed the data with an ANW-OSEM (attenuation and normalization weighted ordered subsets expectation maximization) algorithm as well for comparison purposes (invIMPSF ANWOSEM and varIMPSF ANW-OSEM) and evaluated the different reconstructions using up to 15 iterations and 21 subsets for both scanners. All the reconstructions used a $336 \times 336 \times 109$ image grid, with a $2 \mathrm{~mm}$ voxel.

2.7.1. Point source array. Acquired data from a single point source array with $15 \times 8$ (Hi-Rez-, $\sim 29$ $\mathrm{MBq}, 13$ min acquisition, $\sim 90$ million prompts ) and $14 \times 9$ (TrueV-, $\sim 10 \mathrm{MBq}, 1 \mathrm{~h}$ acquisition, $\sim 137$ million prompts) $\left[{ }^{18} \mathrm{~F}\right]$ printed sources were used to assess the potential resolution improvements of the different resolution modelling methods. Resolution recovery was assessed qualitatively by looking at point source profiles as well as quantitatively by calculating the FWHM for the different methods.

2.7.2. NEMA image quality phantom. To evaluate the bias-variance characteristics of the different algorithms the NEMA IEC image quality phantom set was used as defined in the NU 2-2001 PET performance measurements (Daube-Witherspoon et al 2002). The background in the phantom and the six spheres $\left(10,13,17,22,28\right.$ and $37 \mathrm{~mm}$ in diameter) were filled with $50 \mathrm{MBq}$ of $\left[{ }^{18} \mathrm{~F}\right]$ with a $4: 1$ sphere-to-background activity concentration. On the Hi-Rez the phantom was scanned without the lung insert (350 Mcounts, 60-minute list-mode acquisition). On the TrueV the largest 2 spheres were filled with non-radioactive solution (water) to simulate cold spot regions in the body (140 Mcounts, 9 min acquisition). Samples were taken from the spheres and the background, measured in a well counter and decay, dead time, background and volume corrected. Data were analyzed qualitatively as well as quantitatively. Six circular regions of interest (ROIs) of similar diameter to the spheres were drawn on the transaxial CT image. For each of the 6 spheres 12 ROIs in total of the same diameter were drawn on the background in the plane that was used for the sphere analysis as well as in planes $\pm 1 \mathrm{~cm}$ and $\pm 2 \mathrm{~cm}$ from that one ( 60 ROIs /per sphere size over the 5 planes). For the bias and the variance assessment 2 figures of merit where calculated and referred to as the contrast recovery coefficient (CRC) (equation 6) and image roughness (IR) (eq7).

$$
C R C_{h, j}=\frac{\left(C_{h, j} / C_{b g, j}-1\right)}{\left(a_{h} / a_{b g}-1\right)} \cdot 100 \%
$$




$$
I R_{j, k}=\frac{\sqrt{\sum_{p=1}^{P_{g g}, j}\left(C_{b g, j, k, p}-C_{b g, j, k}\right)^{2} /\left(P_{b g, j}-1\right)}}{C_{b g, j, k}} ; I R_{j}=\frac{\sum_{k=1}^{N_{b g}} I R_{j, k}}{N_{b g}}, \quad N_{b g}=60
$$

where $C_{h, j}$ is the mean reconstructed activity concentration (AC) for the $j$ th hot sphere, $C_{b g, j}$ is the mean reconstructed $\mathrm{AC}$ for the $j$ th background sphere averaged over the 60 ROIs of equal size, $a_{h, j}$ and $a_{b g, j}$ are the measured ACs in the hot spheres and the background, $C_{b g, j, k}$ is the mean AC for the $j$ th background sphere size and the $k$ th ROI, $C_{b g, j, k, p}$ is the reconstructed AC for the $p$ th image voxel within the $k$ th ROI for $j$ th background sphere and $P_{b g, j}$ is the number of pixels in the $j$ th background sphere.

2.7.3. Cologne resolution phantom. The Cologne resolution phantom (Max-Planck Institute, Cologne, Germany) was scanned on the TrueV PET/CT to assess the resolution improvements by visual evaluation of the images and profiles. The phantom has drilled holes in groups of $5 \times 5$ with a hole diameter of 5, 4, 3 and $2 \mathrm{~mm}$ ( 325 holes in total) and centre to centre spacing equal to double the hole diameter in a $28.6 \mathrm{~mm}$ thick plexi-glass slice. The insert is stacked between additional plexi-glass slices. On one side a containment disk squeezes the solution in the holes while on the other side a semipermeable membrane holds the water within the holes letting the air escape. The phantom was filled with $75 \mathrm{MBq}$ of fluorine-18 and positioned in the scanner with the centre of the phantom coinciding with the centre of the FOV. A 60-minute scan was acquired in list-mode and $\sim 140$ million counts were collected.

2.7.4. $\left[{ }^{11} \mathrm{C}\right]$ ASO abdominal PET/CT study. A single scan from a study examining the bio-distribution of a carbon-11 labelled antisense oligonucleotide was used to compare observed resolution improvements with the different reconstruction methods on the Hi-Rez (Saleem et a/ 2009). With this tracer high uptake is observed within the kidney cortex. The patient was administered with $441 \mathrm{MBq}$ of $\left[{ }^{11} \mathrm{C}\right]$ ASO and list-mode data were acquired over a 95 minute period using the Hi-Rez PET/CT. Almost 60 million prompts were collected and histogrammed into a static sinogram.

2.7.5. [18-F] FLT abdominal PET/CT study. A single oncology [18F]-FLT (fluoro-3'-deoxy-3'-Lfluorothymidine) dataset was used to assess the different methods on the TrueV PET/CT. The patient was administered with $332 \mathrm{MBq}$ of $\left[{ }^{18} \mathrm{~F}\right]-\mathrm{FLT}$ and list mode data ( 2.3 billion prompts ) were acquired over a $62 \mathrm{~min}$ period on the TrueV PET/CT. The list mode data were histogrammed in a static sinogram and reconstructed transverse, coronal and sagittal images from all the methods were qualitatively assessed.
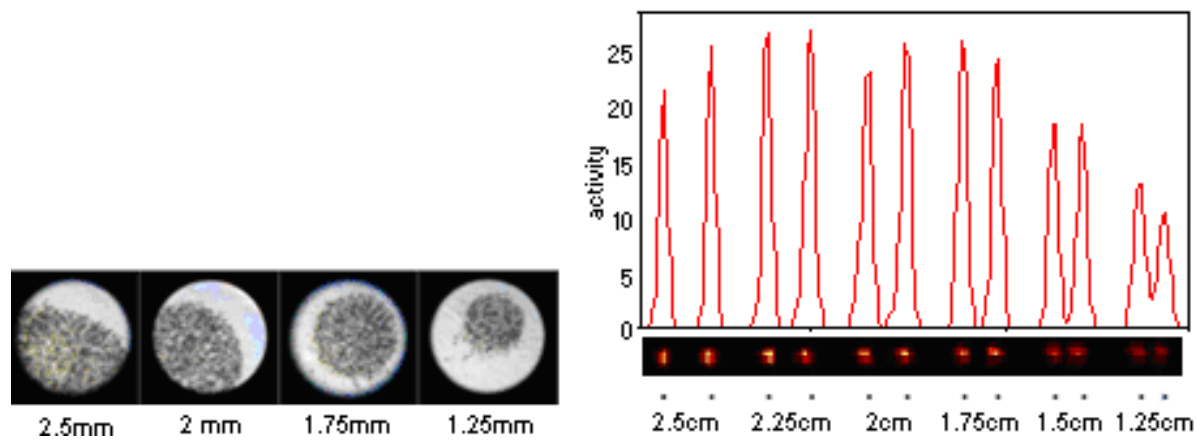

Figure 6. Microscope images taken from printed point sources of different diameter $(1.25 \mathrm{~mm}, 1.75 \mathrm{~mm}, 2 \mathrm{~mm}$, and $2.5 \mathrm{~mm})$ after being re-printed 7 times (left). Point spread function profiles of varying diameter and spacing (right). When the spacing of the sources is too close, the distribution tails start to overlap. 


\section{Results}

\subsection{Derivation of a parameterized PSF on the Hi-Rez and TrueV PET/CT}

3.1.1. Optimization of the point source array. Figure 6 shows the profiles from an axial slice through a reconstructed volume with the point sources spaced at decreasing distances toward the edge of the FOV. As the distance between the sources decreases the PSF tails start to overlap. Spacing the sources evenly at intervals of $1.5 \mathrm{~cm}$ was found to be sufficient to differentiate adjacent PSFs. Microscope enlarged images showed ink droplets being printed outside the point source boundaries for sources smaller than $2 \mathrm{~mm}$ and after re-printing the array 7 times (Figure 6). Consequently a conservative approach of using $2 \mathrm{~cm}$ isotropic spacing of $2 \mathrm{~mm}$ diameter sources was employed for all subsequent scans. This optimization was done on the Hi-Rez but the same array configuration was also applied on the TrueV.
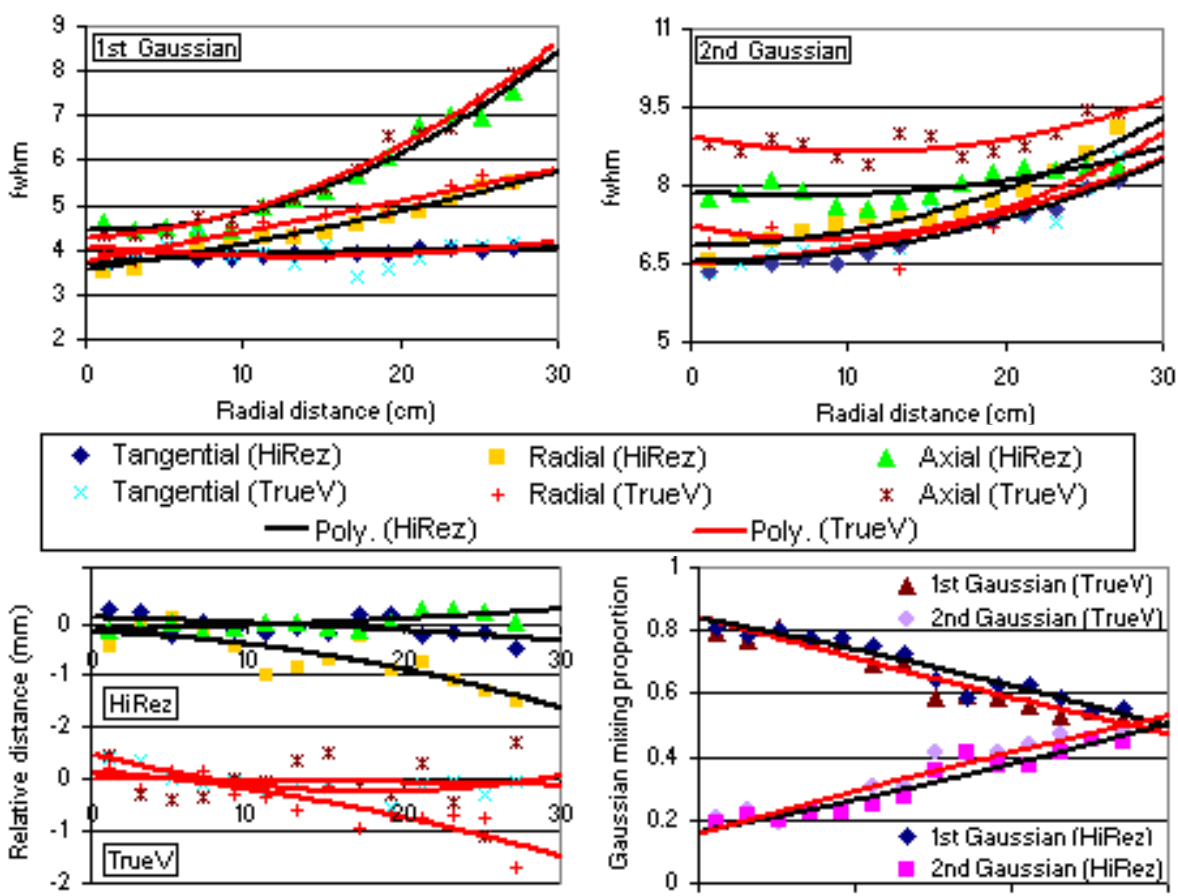

Radial distance (cm)

Axial (HiRez)

* Axial (TrueV)

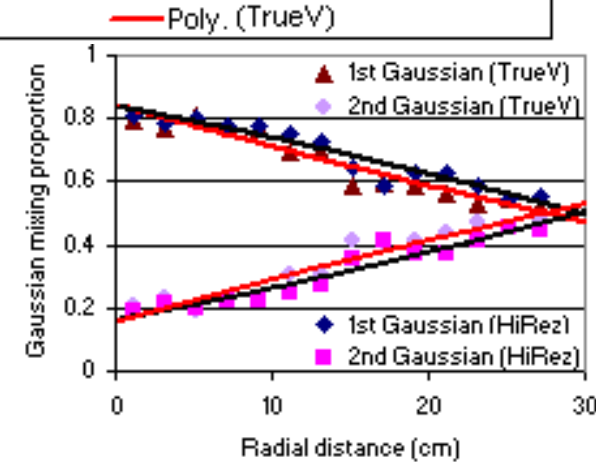

Figure 7 Top: Radial, tangential and axial fwhm for the first (left) and second (right) 3-D Gaussian component as a function of the radial distance from the centre of the FOV for one radial line of point sources $(\mathrm{z}=0)$ for the Hi-Rez and the TrueV. Bottom: Difference (counted in number of voxels) between the $2^{\text {nd }}$ and the $1^{\text {st }}$ Gaussian mean parameters (radial, tangential and axial) as a function of the radial distance (left) and mixing proportions between the 3-D Gaussian distributions for both scanners as a function of the radial distance (right).

3.1.2. PSF parameter dependence on radial position. In figure 7 , the fitted parameters for one row of point sources (14 sources) axially centred in the scanner, are shown both for the Hi-Rez and the TrueV. The $1^{\text {st }}$ Gaussian distribution accounts for the main PSF while the $2^{\text {nd }}$ one with wider distribution account for the tails in the distribution. The axial component of the $1^{\text {st }}$ Gaussian appears to introduce the highest blurring followed by the radial and the tangential components (figure 7 top left). At increasing radial distance there is a synergistic effect, in both scanners, between the axial undersampling induced blurring and the parallax error resulting in the increased resolution degradation in the axial direction compared to the radial and tangential one. Looking at the relative mean for the 2 Gaussian distributions we can see that axially and tangentially there is no relative shift between for all radial positions meaning that the overall distribution is symmetric along these 2 directions. In contrast, the means are displaced radially, relating to a skewed asymmetric distribution. This is possibly as a consequence of parallax errors, with a degree of asymmetry increasing with radial position (figure 7 bottom left). As the radial position increases, the $2^{\text {nd }}$ Gaussian distribution accounts for an increasing proportion over the $1^{\text {st }}$ Gaussian distribution in the overall PSF function (figure 7 bottom right), resulting in PSFs with larger tails and asymmetric in the radial direction. The trend is similar both for the Hi-Rez and the TrueV. 

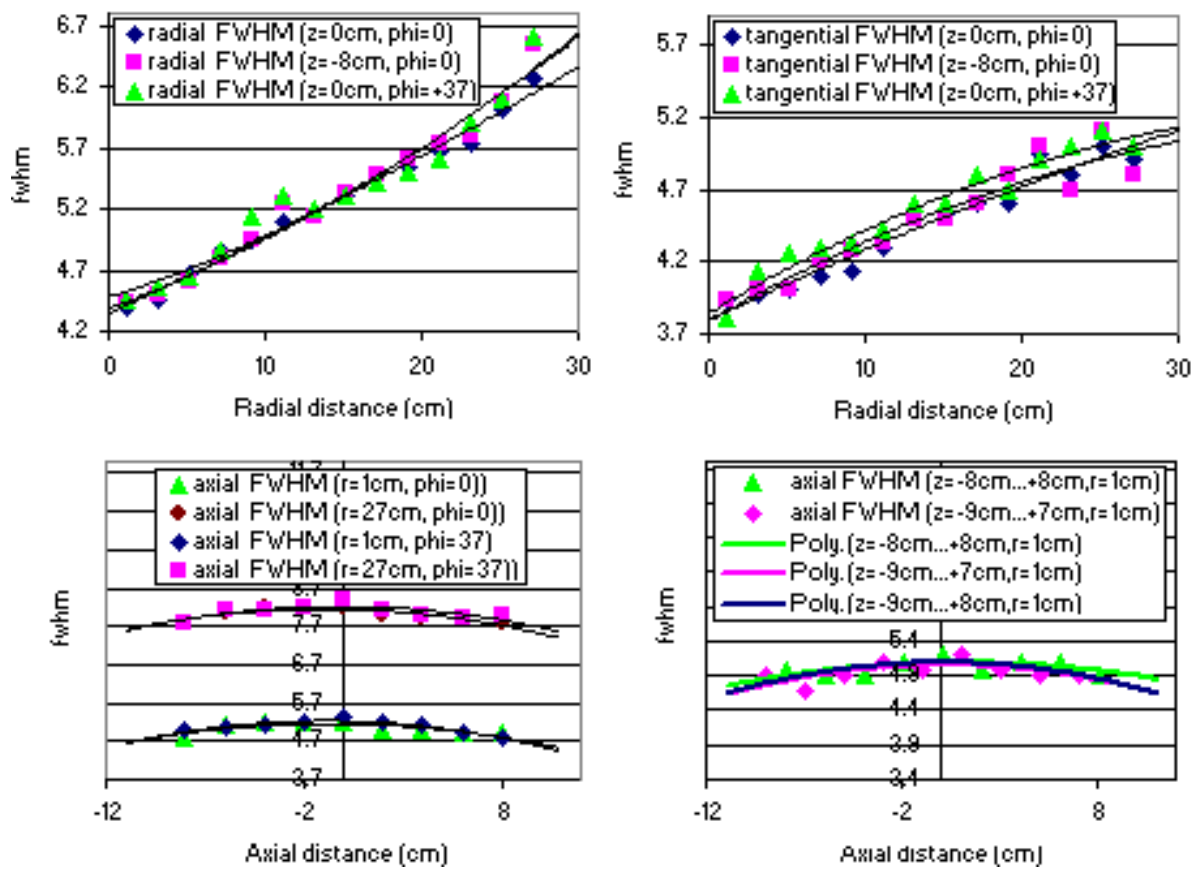

Figure 8 .Top: Radial (left) and tangential (right) FWHM (TrueV) for different axial, radial and angular positions in the FOV as a function of radial distance. Bottom: Axial FWHM at different radial positions and azimuthal angles as a function of axial distance (left) and axial FWHM versus axial distance for 2 different axial positions of the array and the polynomial fit using data from each position individually or from their respective combination (right). Data were reconstructed using the in-house image reconstruction with no resolution modelling.

3.1.3. PSF axial dependency and parameter reproducibility from a single scan. Figure 8 (top left and right) shows the FWHM of the radial and tangential PSF components on the TrueV as a function of the radial distance from the centre of the FOV at 2 different axial positions $(\mathrm{z}=0 \mathrm{~cm}, \mathrm{z}=-8 \mathrm{~cm})$ and at 2 different azimuthal angles $\left(\phi=0^{\circ}, \phi=37^{\circ}\right)$. As expected the angle-dependent crystal penetration as a function of the radial distance results in an increasing blurring both in the radial and tangential directions. Looking at the system response at the centre and at the edge of the axial FOV, the radial and the tangential components of the PSF are practically axially symmetric. The same also applies for the system response at the 2 different azimuthal angles effectively making the radial and tangential components rotationally symmetric, omitting the need for additional acquisitions at different azimuthal angles. Similar results where taken on the Hi-Rez with minimal axial dependency mainly due to the axially dependent transaxial FOV. For the axial component (Fig.8 bottom left) when looking at the FWHM at the centre and the edge of the transaxial FOV $(r=1 \mathrm{~cm}, r=27 \mathrm{~cm})$ it also varies as a function of the radial distance. In addition, the axial blurring is also variant in the axial direction but rotationally symmetric when compared to the axial response from a different azimuthal angle $\left(\phi=37^{\circ}\right)$. The axial blurring reduces slightly towards the edge of the axial FOV but this axial dependency is very small compared to the radial one. Importantly though the axial component is rotationally symmetric, similar to the radial and tangential ones, and as such only a single 2-dimensional surface through the 3-D image space needs to be sampled. As all the PSF components vary slowly within this surface, without any sudden discontinuities, the coarse sampling using a single point source array can capture the smoothly varying blurring properties of the scanner. This is demonstrated in the bottom right graph from figure 8 where the axial FWHM taken from 2 different axial positions of the array $(9+9$ point sources) shifted by $1 \mathrm{~cm}$ with respect to each other, is plotted as a function of axial distance. The polynomial fit using only the 9 point sources from each individual scan as well as using 18 point sources from both scans effectively doubling the sampling is also shown. Although it is expected that in each plane the axial FWHM depends on the span and the number of segments contributing to that plane, the fit obtained from a single scan (9 point sources) is very similar to the one using a finer sampling by combining the 2 scans. As this axial dependency of the axial blurring is very subtle and in order to reduce the storage of the PSF it can be approximated by an axially invariant axial blurring component with minimal impact on the model accuracy. 


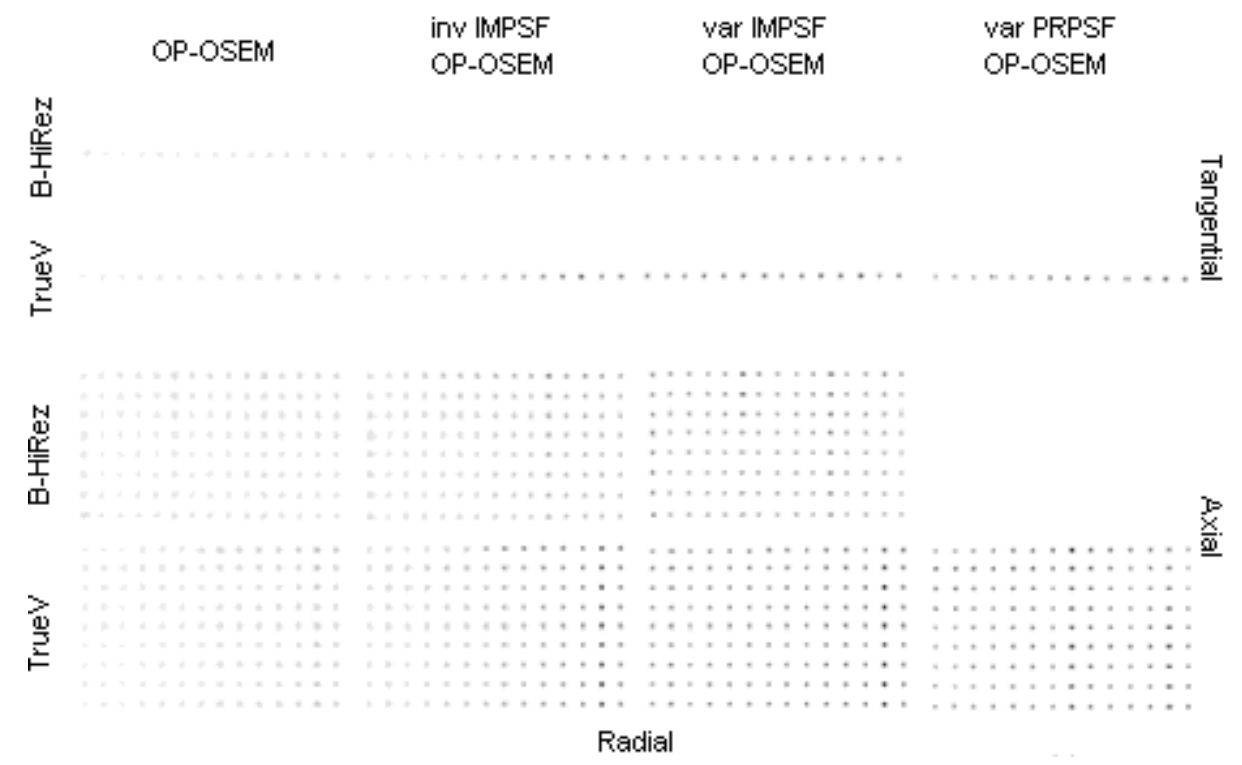

Figure 9. Transaxial $(\mathrm{z}=0)$ and horizontal sections through the 3-D image space for point source data reconstructed with OPOSEM, with: no resolution modelling (column a: OP-OSEM); a spatially invariant image based kernel (column b: invIMPSF OPOSEM); with the measured space variant image based PSF model (column c: varIMPSF OP-OSEM) and with the measured space variant projecton based PSF model (column d: var PRPSF OP-OSEM).
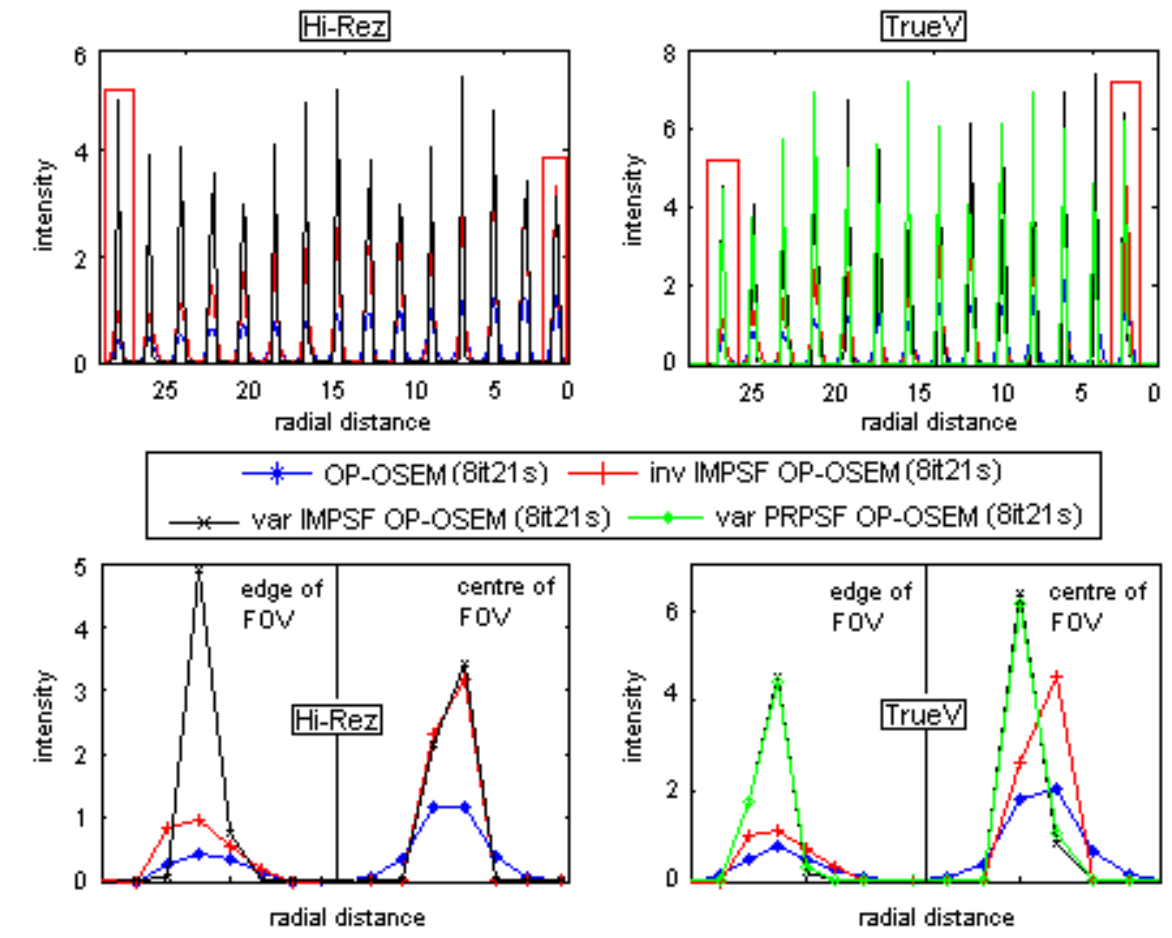

Figure 10. (top row) radial profiles through point source images $(\mathrm{z}=0)$ reconstructed using OP-OSEM, invIMPSF OP-OSEM, varIMPSF OP-OSEM and var PRPSF OP-OSEM for Hi-Rez (left) and TrueV (right). 2 point source at the centre and the edge of the FOV are zoomed in the bottom 2 graphs. Nearly identical profiles are seen both for the variant image based and projection spaced PSF reconstructions.

\subsection{Reconstruction performance evaluation and validation}

3.2.1. $\left.{ }^{18-} \mathrm{F}\right]$ point source data. When no resolution modelling is used (figure 9 ) the counts spread out in all directions with increasing blurring towards the edge of the FOV. This results in asymmetric distributions with decreasing amplitude looking as shown by line profiles through the point sources (Figure 10). The use of the invIMPSF method results in a dramatic increase in the recovered activity, particularly close to the centre of the FOV (Figure 9) as the used kernel matches the scanner's blurring in this region (4.3mm FWHM). Away from the centre though the there is still clear blurring of the point 
sources due to the discrepancy between the invariant kernel and the true kernel. With the spatially variant image based resolution modelling and the projection space resolution modelling resolution improvements are observed, both towards the edge of the FOV as well as close to the centre. Comparing these two algorithms (varIMPSF OP-PSEM and varPRPSF OP-OSEM) at the same iterations and subsets on the TrueV we get almost identical recovered profiles at the 2 extreme positions in the FOV (figure 10. bottom right) which reflects the very similar performance of these algorithms and that the resolution in now limited by the image voxel sizes. This limited sampling of the image results in observed periodic variability in the maximum point source intensity with both scanners and both algorithms.
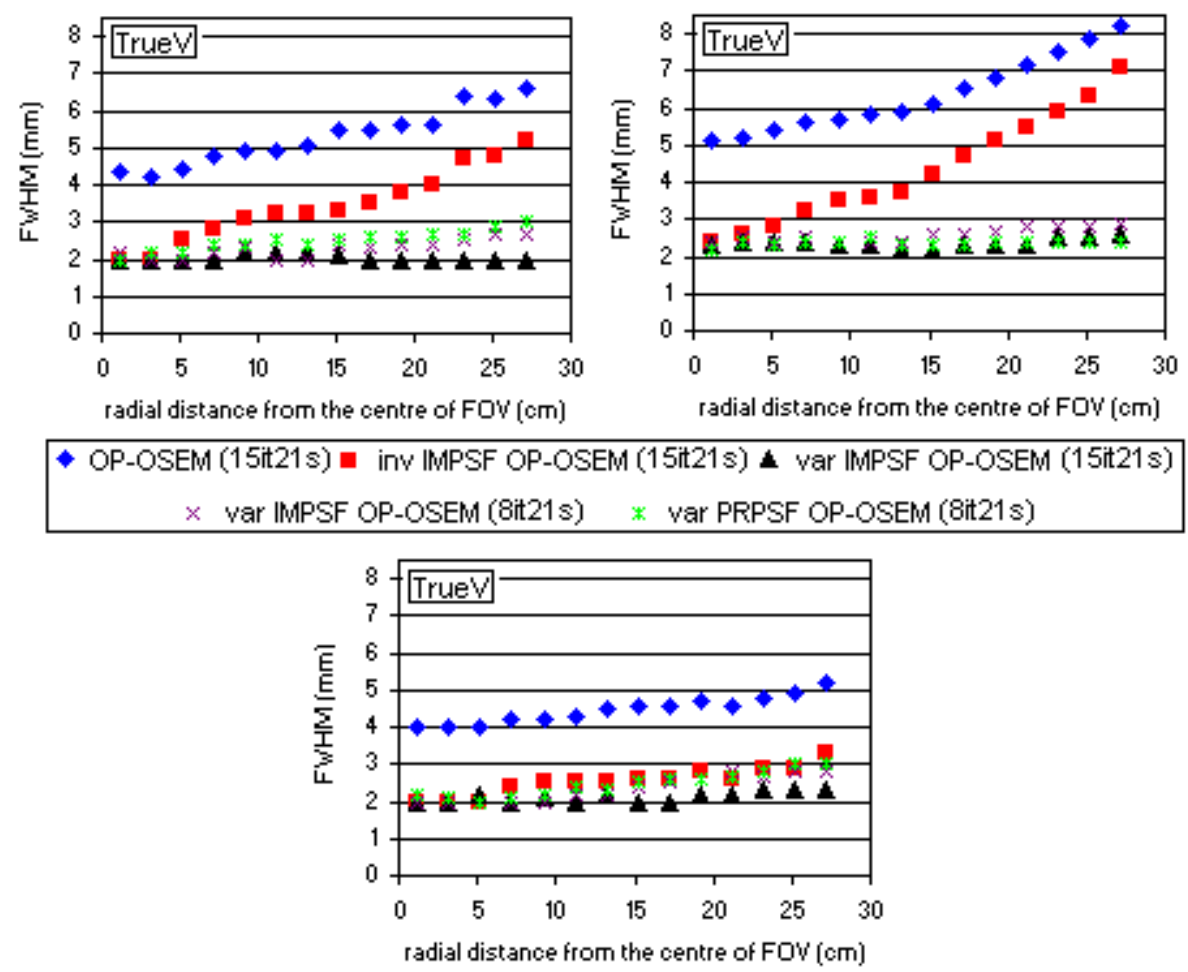

Figure. 11. Radial (top left), axial (top right) and tangential (bottom) FWHM ( $\mathrm{z}=0$ ) on the TrueV PET/CT as a function of radial distance for the all the reconstruction methods.

Figure 11 quantifies the resolution improvements obtained by including resolution modelling within the reconstruction. Using OP-OSEM with no resolution model the axial resolution is the worst, followed by the radial and tangential. Using invIMPSF OP-OSEM the resolution is improved in all directions by almost $2 \mathrm{~mm}$ in the centre of the FOV while at the edge the improvement is slightly less pronounced. When the varIMPSF model is used, an almost uniform resolution of $2 \mathrm{~mm}$ is achieved in the radial, axial and tangential directions. Comparing the varIMPSF against the varPRPSF at the same number of iterations (8 iterations 21 subsets) we see very similar resolution improvements with the varPRPSF achieving slightly better axial resolution towards the edge of the FOV while the varIMPSF achieves marginally better radial resolution towards the edge of the radial FOV. Finally the resolution (axial, radial and tangential) after 8 iterations is lower than that achieved after 15 iteration, as 8 iterations are insufficient for convergence to be reached and with the effect being greater for points towards the edge of the FOV.

3.2.2. NEMA IEC image quality phantom. Figure 12 shows reconstructed images from the NEMA IEC image quality phantom both for the Hi-Rez (high statistics scan) and the TrueV (low statistics scan). Qualitatively the PSF reconstructions can handle the noise better when looking at the uniform background regions with larger improvements when using the variant PSF model. Improvements are also apparent in the sphere-to-background contrast especially in the smallest sphere (10mm). Looking at the varIMPSF reconstruction from either scanner as well as the varPRPSF reconstruction, ringing (or Gibbs) artefacts start to appear at the boundaries of the phantom with the Gibbs artefacts slightly more intense in the varPRPSF reconstruction. Figure 13 shows the quantitative analysis on the phantom data where the $\mathrm{CRC}$ for the $10 \mathrm{~mm}$ and $22 \mathrm{~mm}$ spheres is plotted against the IR for increasing number of 
iterations. For the smallest sphere $(10 \mathrm{~mm})$ the addition of a resolution model in the reconstruction improves the hot sphere CR as well as the IR in both scanners while for the $22 \mathrm{~mm}$ sphere the contrast improvements are more subtle. The inclusion of the PSF model in the reconstruction on the other hand changes the convergence characteristics of the algorithms. In particular the value of CR obtained with the non-PSF reconstruction stabilises to a constant value after 4-5 iterations for the high statistic HiRez data set while for both PSF reconstructions the CR value for the $10 \mathrm{~mm}$ sphere continues to increase even after 15 iterations.

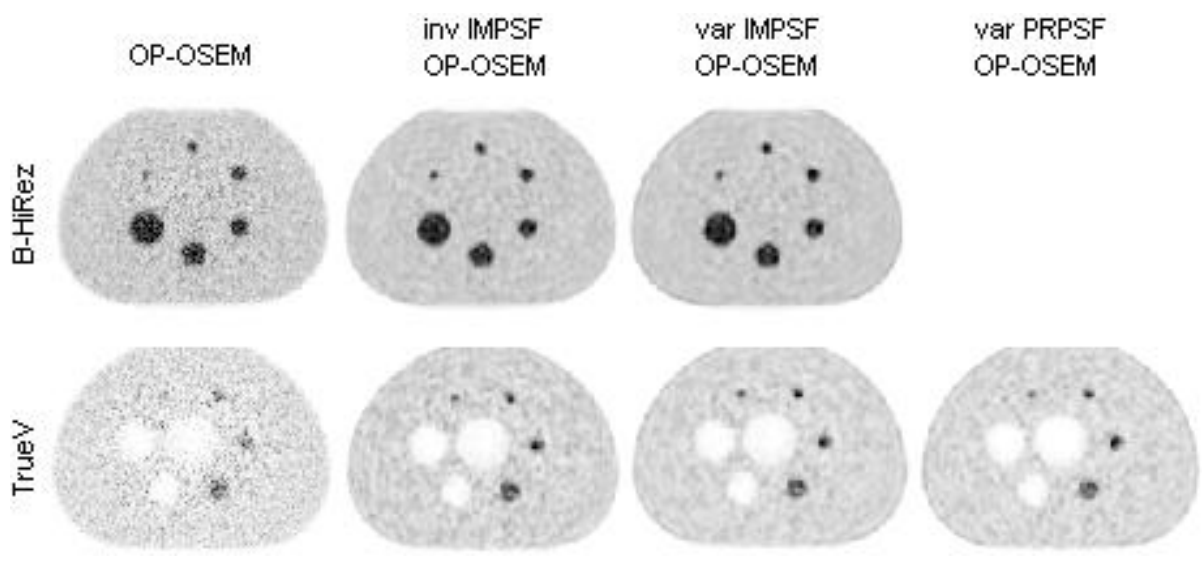

Figure 12. Reconstructed images using the NEMA phantom on the Hi-Rez (top) and the TrueV (bottom) compared at the same noise level. The images with the variant PSF appear to have the best contrast with the penalty of Gibbs artifacts.

On the TrueV the OP-OSEM based reconstructions outperform the ANW-OSEM based ones with lower IR at the same CR level, as the data on the TrueV are noisier ( $\sim 140$ million counts) than that on the Hi-Rez ( $\sim 350$ million counts) and OP-OSEM can provide a better handling of statistical noise. Comparing the image based method against the projection based method for the $10 \mathrm{~mm}$ and the $22 \mathrm{~mm}$ spheres, the varIMPSF and the varPRPSF algorithms achieved similar CRC at the same IR level with the varIMPSF reconstruction giving slightly better results.
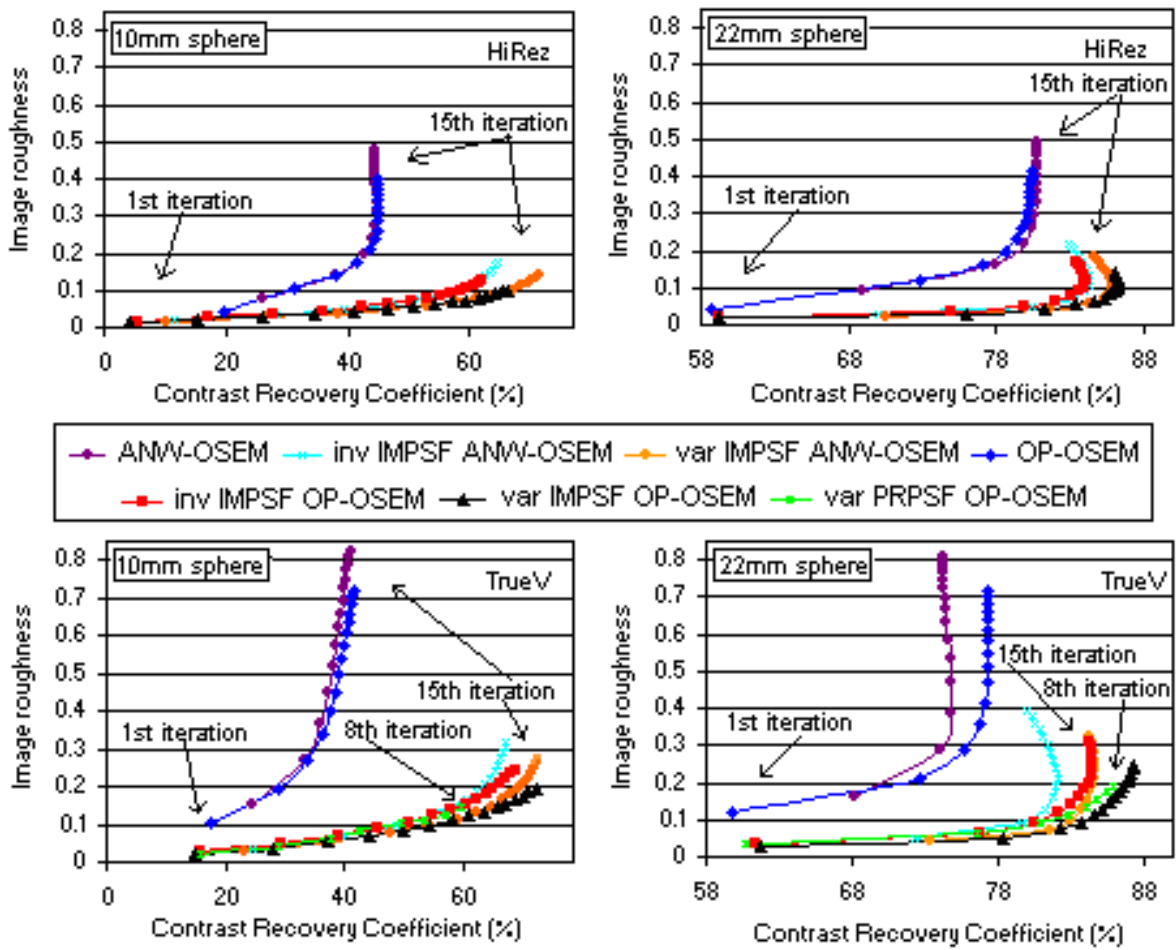

Figure 13. Contrast recovery coefficient and image roughness trade-off as a function of iteration as calculated using the NEMA IEC phantom scanned on the Hi-Rez (top row) and the TrueV (bottom row) for a $10 \mathrm{~mm}$ (left column) and a $22 \mathrm{~mm}$ (right column) spheres. 
3.2.3. Cologne resolution phantom. As shown in Figure 14 using no resolution modelling the $4 \mathrm{~mm}$ and $5 \mathrm{~mm}$ tubes can easily be distinguished from each other but appear noisy while the $2 \mathrm{~mm}$ tubes are practically inseparable. Inclusion of a resolution model improves the resolution in the $4 \mathrm{~mm}$ and $5 \mathrm{~mm}$ tubes. Conversely the separation of tubes for the $2 \mathrm{~mm}$ and $3 \mathrm{~mm}$ tubes has deteriorated in the invIMPSF, varIMPSF and the varPRPSF reconstructions. As the number of iterations in the PRPSF reconstruction in the scanner is limited to 8 our inv IMPSF and var IMPSF reconstructions were also limited to the same number of iterations. As a result is difficult to resolve the smaller $2 \mathrm{~mm}$ and $3 \mathrm{~mm}$ tubes with the resolution recovery reconstructions which will most likely require more iterations due to slower convergence. In addition at the edge of the block of tubes, particularly for the outer blocks Gibbs ans possible aliasing artefacts are visible. Looking at the differences between the variant and the invariant PSF based reconstructions these are very subtle and are mainly located in the $4 \mathrm{~mm}$ and $5 \mathrm{~mm}$ spheres with the largest radial displacement from the centre of the phantom. Comparing the image based and projection based PSF reconstructions qualitatively no significant difference can be seen, with similar resolution performance in the $4 \mathrm{~mm}$ and $5 \mathrm{~mm}$ spheres.

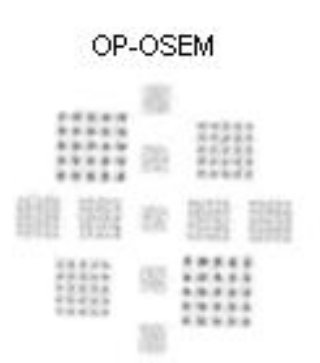

(a)

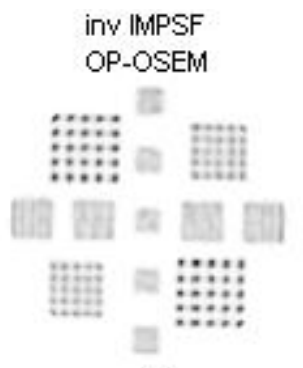

(b)

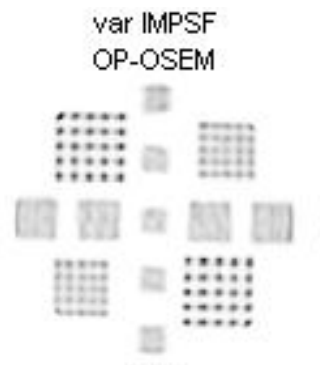

(c)

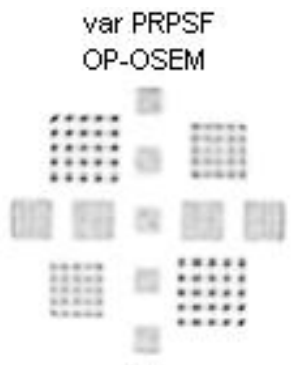

(d)

Figure 14. Reconstructed images from the Cologne phantom using OP-OSEM (a), invIMPSF OP-OSEM (b) varIMPSF OP-OSEM (c) and var PRPSF OP-OSEM, The PSF methods show improved resolution especially on the $4 \mathrm{~mm}$ and $5 \mathrm{~mm}$ spheres with a marginally better resolution for the variant PSF algorithms.

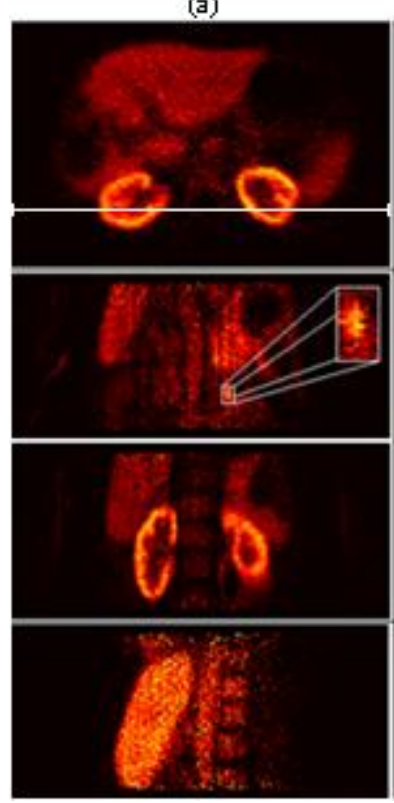

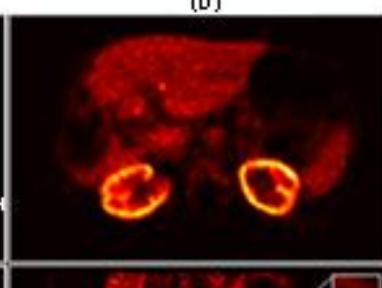
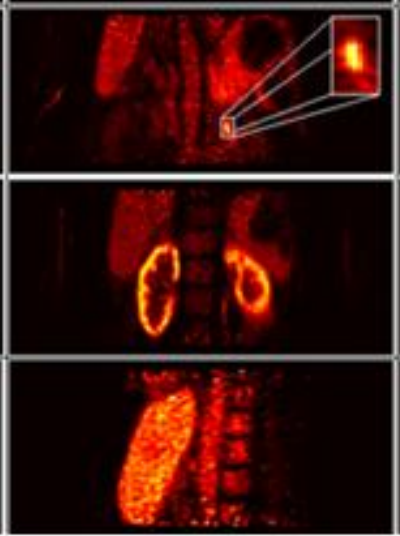

(c)

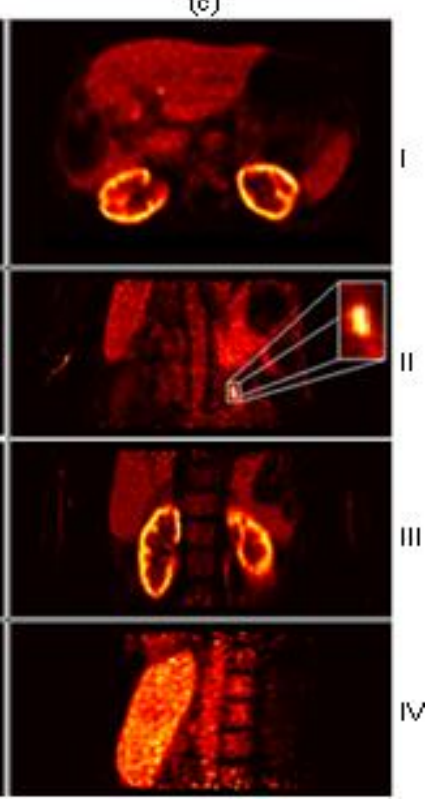

Figure 15. Transverse (I), coronal (II, III) and sagittal (IV) images from a $\left[{ }^{11} \mathrm{C}\right]$-ASO study reconstructed with OP-OSEM (a), invIMPSF OP-OSEM (b) and varIMPSF OP-OSEM (c).

3.2.4. $\left[^{11}\right.$ CJ-ASO abdominal PET/CT study. Transverse, coronal and sagittal images from an abdominal study with $\left[{ }^{11} \mathrm{C}\right]$-ASO on the Hi-Rez are shown in figure 15 . Visual inspection shows significant variance reduction especially in the liver region in the transverse images using the variant PSF reconstruction. Looking at a high activity concentration structure located close to the aorta in the coronal images, increased contrast is also seen with the improvements of the variant PSF methods over the invariant method. These differences are more pronounced close to the edge of the FOV as observed within hand veins close to the injection site. In terms of resolution a much better delineation of the 
kidney cortex is seen in the transverse and coronal images with an improved resolution recovery of the variant PSF over the invariant. This qualitative assessment confirms the quantitative bias, variance and resolution results obtained with the NEMA phantom as well as with the point sources. These findinds are also confirmed looking at profile along the transverse images (Figure 17a)

3.2.5. ${ }^{18}$ F]FLT abdominal PET/CT study. On the TrueV, data from an $\left[{ }^{18} \mathrm{~F}\right]-\mathrm{FLT}$ scan were used to qualitatively assess the different reconstruction methods. Figure 16 shows the transverse, coronal and sagittal maximum intensity projection images from such a dataset. The resolution modelling methods show a significant variance reduction, especially at the edge of the axial FOV as the reduced sensitivity causes an increase in statistical noise. The spatially variant resolution modelling methods (varIMPSF and varPRPSF) perform better compared to the invIMPSF method with better delineation of activity within the ribs and within tubing associate with the injection site. This is consistent with the phantom data with these regions located away from the centre of the FOV. Increased contrast is also seen with background noise suppression and better organ delineation. Comparing the varIMPSF against the varPRPSF again no significant difference can be seen with both methods reconstructing images of high resolution and SNR. This also confirmed looking at the profiles along the transverse images (Figure $17 b)$.

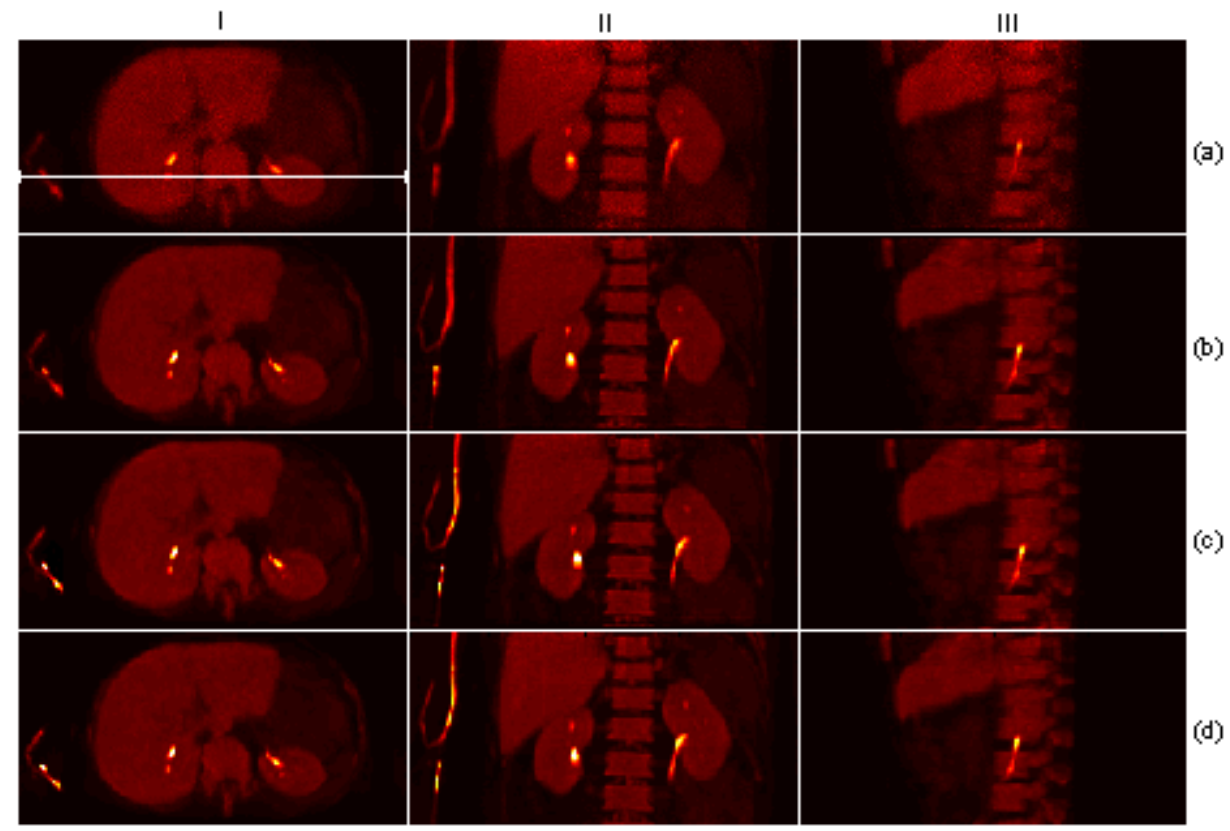

Figure 16. Transverse (I), coronal (II) and sagittal (III) maximum intensity projection images from a $\left[{ }^{18} \mathrm{~F}\right]$-FLT study reconstructed with no resolution modelling (a), with an invIMPSF resolution model (b), with a varIMPSF model (c) and with a var PRPSF model (d).

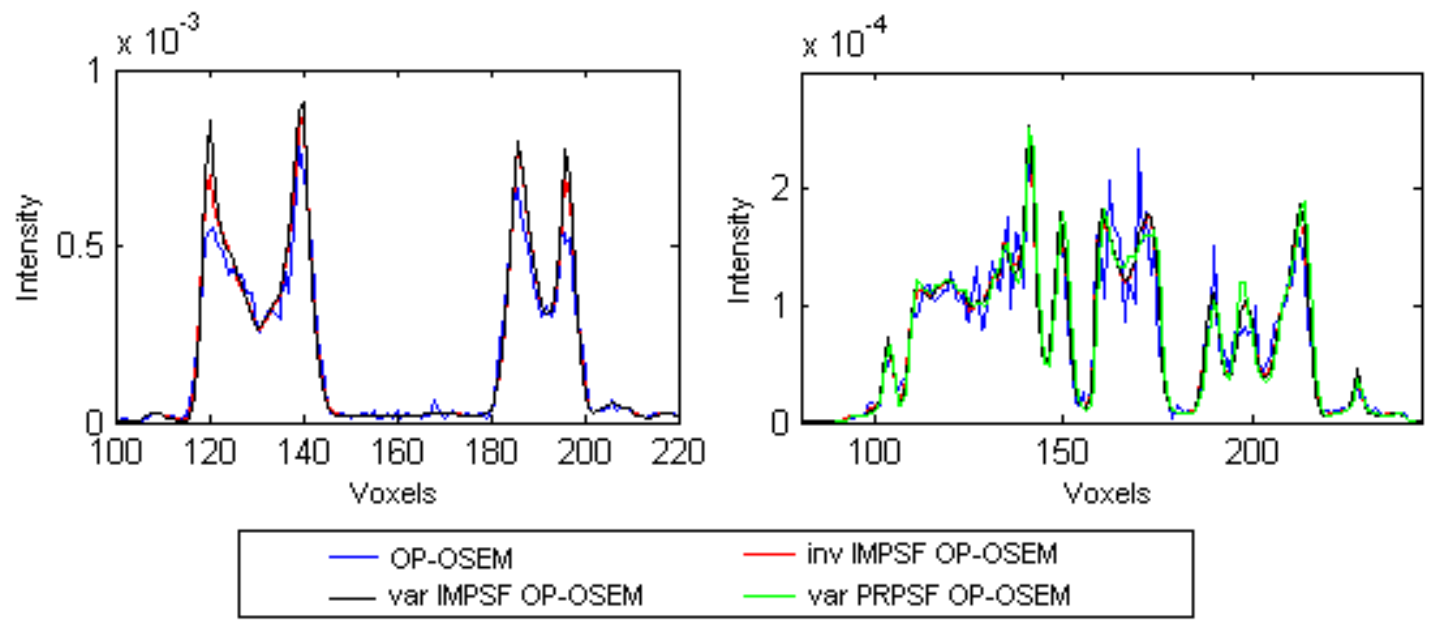

Figure 17 Profiles through the transverse $\left[{ }^{11} \mathrm{C}\right]$-ASO (left) and $\left[{ }^{18} \mathrm{~F}\right]-\mathrm{FLT}$ (right) patient images for the different image reconstruction algorithms 


\section{Discussion}

Recent developments in PET imaging both in terms of software and hardware have enabled the acquisition and reconstruction of quantitative and high resolution clinical images. With most of the new generation clinical scanners taking into account the system's response during the image reconstruction process, there is a need for developing practical as well as cost and time efficient methodologies for evaluating the scanner's blurring properties. In this work we proposed, and optimised a novel methodology to characterize the image based spatially variant PSF, and implemented this methodology on the Biograph 6 B-Hi-Rez and TruePoint TrueV PET/CT scanners. Furthermore we evaluated the impact of resolution modelling using these measurements on a number of phantom and clinical studies. Of note, for the first time, we present a direct comparison between image based and projection based PSF resolution modelling reconstructions using the same datasets in order to quantity any loss of performance with the image based approach. Based on our findings, not only the image based resolution modelling method can very accurately approximate the projection space resolution modelling method but at the same time scanning a single point source array is sufficient to capture the spatially variant system response in the form of image based kernels. Using a single scan also to derive the blurring kernels greatly facilitates investigating other resolution degradation effects like detector pile-up from increased count rate as well as measuring isotope specific kernels especially from shortlived isotopes like $\left[{ }^{11} \mathrm{C}\right]$ and even $\left[{ }^{15} \mathrm{O}\right]$, not previously possible, using a single point source.

\subsection{Experimental design and methodology}

From a methodology point of view using a printed point source array enabled us to simultaneously scan 126 point sources and measure the changing shape of the PSF kernel. By printing the array multiple times we acquired data with high statistics providing good quality fits during parameterization of the PSFs. Also for the Hi-Rez and TrueV scanners the parameters describing the PSF kernels varied sufficiently slowly across the FOV that $2 \mathrm{~cm}$ spacing was adequate to characterise these variations. This is likely to be the case for other cameras, however if not then finer sampling can be obtained by moving the array in the axial and radial directions as was done with the Hi-Rez data.

Using the Perspex phantom for positioning the point sources provided an excellent alternative to previously used robotic systems, obviating the need for accurate alignment of each source independently, once a single line of sources in the array was aligned. On the Hi-Rez multiple acquisitions were performed to finely sample spatial variations in the PSF kernels which provided us with knowledge regarding sudden discontinuities in the system's response along the radial and axial directions.

On the TrueV PET/CT we systematically exploited the symmetric properties of the PSF in image space to optimize the PSF model and reduce its dimensionality. This allowed estimation of the PSF model parameters from a single array scan. As we modelled the PSFs in image space where there were no sudden changes in the system's response from the block edge effect (as opposed to projection space modelling), the 14 radial points in the array provided sufficient sampling to characterize the radial component. In the axial direction the axial compression scheme used in projection space (5 or 6 LORs combined per plane) and the ring gaps destroy the axial translation symmetry in image space as well but the 9 axial point sources per radial position in the array are sufficient to approximate the variation in the axial response (figure 8) as it varies more slowly than the radial response. To further reduce statistical noise the array can be scanned for longer periods as there is no need for acquiring data at multiple positions.

It was not necessary to apply filtering to the data prior to parameterization as done by Rapisarda $e t$ al (2010) due to acquiring high counting statistics data, resulting in reduced noise in the reconstructed PSFs. This also preserved the high frequency components of the measured response. Furthermore the polynomial fitting that was applied to the PSF parameters to estimate them at the remaining unmeasured positions in the FOV, provided a natural regularization to the measured response. For the model fitting we chose to parameterize the data as a weighted sum of 3-dimensional Gaussian distributions as opposed to using one Gaussian distribution combined from 2 half Gaussian functions (Panin et a/ 2006, Rapisarda et al 2010). This allowed us to model the long tails observed on the PSF data which can't be accounted for when only one Gaussian is used (Sureau et al 2008 , Cloquet et al 2010). Other models could also be considered like the asymmetric modified Pearson model (Cloquet $e t$ a/ 2010). One important aspect of the parameterization is that the fitted parameters and their positional variation are unique to our forward and back projectors which were based on an implementation of the Siddon algorithm (Siddon 1985). Using other methods which more accurately model the geometric 
component of the system matrix, will result in a different set of parameters, with less resolution blurring effects left to be modelled by the blurring components of the system matrix.

Looking at the PSF parameterization across the 2 scanners, the radial and tangential FWHM appears to be very similar. This is expected mainly due to the almost identical crystal ring diameter and crystal dimensions as well as due to the fact that the response in image space is an average of any detector specific blurring and as such any difference in projection space are averaged in image space. On the other hand the axial resolution was found to be slightly higher on the TrueV looking at the FWHM of the $2^{\text {nd }}$ Gaussian (figure 7) (Jakoby et a/ 2006, 2009). This could possibly be attributed to the addition of a $4^{\text {th }}$ block ring and an extra segment on the TrueV, allowing LORs with higher co-polar angles to be detected thus creating longer tails in the axial direction of the PSF. Also the barrel shape of the Hi-Rez probably accentuates this difference as it reduces the parallax error in the centre of the axial FOV due to the smaller co-polar angles of the oblique segments.

\subsection{Reconstruction performance evaluation and image versus projection based resolution modelling comparison}

We evaluated the impact of the proposed methodology with resolution modelling reconstruction on both the Hi-Rez and the TrueV PET/CT scanners using phantom datasets (point sources, NEMA phantom, Cologne phantoms) to quantify the improvements as well as clinical datasets $\left(\left[{ }^{11} \mathrm{C}\right]\right.$-ASO and $\left.\left[{ }^{18} \mathrm{~F}\right]-\mathrm{FLT}\right)$ to verify that similar qualitative improvements were observed with real data.

Looking at the reconstructed point sources (figure 9) as well as the 1-D radial profiles (figure 10) we saw similar performance and little difference between the image based and the projection based PSF reconstructions as evaluated for the TrueV scanner. The similarity in these profiles (figure 10 bottom right) demonstrates that the degree of radial asymmetry has been captured by both methods to an almost equal degree of accuracy.

From the analysis of the point sources we measured an almost uniform resolution of $2 \mathrm{~mm}$ throughout the FOV using varIMPSF OP-OSEM with the proposed methodology after 15 iterations. We weren't able to achieve similar resolution improvements (uniform $2 \mathrm{~mm}$ resolution) with the varPRPSF reconstruction as reported by Panin et a/ $(2006,2007)$ due to the scanner's restriction of a maximum of 8 iterations. To accurately compare the methods though, we reconstructed the data using our in house implementation for 8 iterations in order to match the number of iterations used in the scanner's varPRPSF reconstruction. In our hands the varIMPSF reconstruction achieved marginally better radial resolution compared to the varPRPSF one probably due to the $2^{\text {nd }}$ Gaussian distribution providing improved tail fitting. On the other hand superior axial resolution was achieved by the varPRPSF method compared to varIMPSF, which can possibly be attributed to the very accurate modelling of the axial blurring in the manufacturer's implementation. Indeed on the TrueV scanner the modelling and application of the axial blurring is done before the axial compression step for each individual LOR, as opposed to modelling the superimposed axial blurring in a span (Panin et a/2006), which facilitates the use of a depth independent axial blurring (Panin et al 2007). The modelled forward projected data are then axially compressed to match the axial sampling of the measured sinograms which have been axially compressed by the online rebinner. As such it is difficult for the average axial blurring measured by the varIMPSF method to match this exact LOR-by-LOR axial PSF modelling.

Similar resolution improvements, consistent with the point source data, were observed in the Cologne phantom. Using smaller voxels during reconstruction can potentially reduce these sampling effects. Gibbs artefacts were also seen on the NEMA phantom reconstructed both with the varIMPSF and the var PRPSF method (Reader et al 2003, Wiant et a/2009, Jakoby et a/2009, Tong et a/2010, Rapisarda et a/2010). One way to minimize the Gibbs would be to use an underestimated kernel compared to the measured one (Snyder et a/ 1987). Such an approach although would compromise the resolution and SNR improvements could potential eradicate the Gibbs artefacts as also suggested by Sureau et al (2008).

On the clinical datasets using the variant PSF reconstructions over the invariant one, results in small improvements sometimes difficult to be assessed qualitatively. Nevertheless looking at the resolution graphs (Figure 10) we can see improvements in the radial and axial resolution of $1 \mathrm{~mm}$ and $1.6 \mathrm{~mm}$ FWHM respectively at $10 \mathrm{~cm}$ away from the centre where many organs and pathologies of interest are located. Although an almost uniform $2 \mathrm{~mm}$ resolution was measured using point sources, this resolution is difficult to achieve in the clinical images. This could be attributed to the presence of statistical noise compared to the high statistics point source data as well as to the non linear nature of the reconstruction algorithm with an activity distribution-resolution correlation (Mustafovic et a/2004). Furthermore the use of progressively larger kernels moving towards the edge of the FOV creates 
spatially variant resolution convergence. This is probably due to the reconstruction problem becoming more ill-posed at the edge of the FOV compared to the centre (Alessio et al 2010). Premature reconstruction termination will result in a non uniform resolution in addition to the activity distribution dependent resolution in the image.

Looking at the overall performance of the image based and projection based PSF reconstructions very little difference were seen between the methods, which was demonstrated not to be relevant in the clinical situation. As our IMPSF and the manufacturer's PRPSF reconstructions are based on different projectors with distinct implementations, is difficult to conclude whether any of the very slight performance differences observed are due to the image based versus projection based strategies or implementation characteristics of the reconstructions. Indeed the agreement of the two algorithms is remarkable considering the potential implementation differences. With the majority of blurring effects within the detection of events, and with an image based approach only approximating these effects, it could be expected that projection based approaches outperform image based approaches. Nevertheless we observed that the image based approach managed to match if not better in some cases the projection based approach. There are two possible reasons for this. First as both methods use a response parameterization, any benefits from a better modelling of the detector blurring using the projection based approach over the image based approach, would be diluted by the fact that the PSF parameters are further interpolated and extrapolated to calculate the response in every position in the FOV. Secondly the manufacturer's implementation avoided measuring the response near the detector gaps (block edge effect) (Panin et a/ 2006) which is a very laborious task and that probably resulted in modelling an average detector response even in projection space, minimizing the potential benefits of better detector modelling compared to the image based approach.

\section{Conclusion}

In this work we propose a fast and efficient way of measuring the space-variant image based blurring component of the system matrix by optimizing the PSF model and exploiting image space symmetries. The data suggest that scanning a single array of $14 \times 9$ point sources is sufficient to fully characterize the spatially-variant blurring component of the system matrix without significantly compromising the accuracy of the model. We showed the resolution and SNR benefit of such an approach on 2 new generation PET/CT systems: the Biograph $6 \mathrm{Hi}-\mathrm{Rez}$ and the Biograph 6 TruePoint TrueV. Similar resolution and SNR improvements were seen between image based and projection based resolution modelling in a variety of phantom and clinical datasets.

Specific benefits of the proposed approach include:

i) Simultaneous acquisition of multiple point sources (obviating the need for accurate alignment of each source independently, once the reference point in the array is aligned),

ii) Rapid acquisition and characterization of the space-variant PSF. This allows easy measurement of the PSF on a scanner-by-scanner basis or even on a repeated basis to regularly to check for any drift in the resolution properties.

\section{Acknowledgments}

This work has been funded by the Engineering and Physical Sciences Research Council (EPSRC) (grant EP/F028695/1. The authors would like to thank M Casey and B Bendriem from Siemens Molecular Imaging, Knoxville, USA for their support and advice on scanner hardware and image reconstruction software and W Hallett from GSK CIC for providing access to the HD image reconstruction software on the TrueV. We are also grateful to $\mathrm{J}$ Anton-Rodriguez for his physics support and the staff and facilities at the Wolfson molecular imaging centre (WMIC). We would also like to acknowledge clinical researchers I Trigonis and A Saleem responsible for the studies in which the clinical data on the $\left[{ }^{18} \mathrm{~F}\right]$-FLT and $\left[{ }^{11} \mathrm{C}\right]$-ASO (with the associated support from EllyLilly and the UK Pancreatic Society) were acquired. Dr Reader acknowledges the support of the Canada Research Chairs programme.

\section{References}

Alessio A, Kinahan P, Lewellen T, 2006 Modelling and incorporation of system response functions in 3-D whole body PET IEEE Trans. Med Imaging. 25 828-37

Alessio A, Stearns CW, Tong S, Ross SG, Kohlmyer S, Ganin A, Kinahan PE, "Application and Evaluation of a Measured Spatially Variant System Model for PET Image Reconstruction 2010 IEEE Trans. Med. Imaging. 29 3938-49 
Brambilla M, Secco C, Dominietto M, Matheoud R, Sacchetti G, Inglese E 2005 Performance characteristics obtained for a new 3-dimensional Lutetium oxyorthosilicate-based whole-body PET/CT scanner with the National Electrical Association NU 2-2001 standard J. Nucl. Med. 46 2083-91

Cloquet C, Sureau F C, Defrise M, Van Simaeys G, Trotta N and Goldman S 2010 Non-Gaussian space-variant resolution modelling for list-mode reconstruction Phys. Med. Biol. 555045

Daube-Witherspoon M E et al 2002 PET performance measurements using the NEMA NU 2-2001 standard J. Nucl. Med. 43 $1398-409$

De Bernardi E, Mazzoli M, Zito F, Baselli G 2007 Resolution recovery in PET during AWOSEM reconstruction: a performance evaluation study IEEE Trans. Nucl. Sci. 54 (5) 1626-38

Jakoby B W, Bercier Y, Watson C C, Rappoport V, Young J, Bendriem, Townsend D W 2006 Physical Performance and Clinical Workflow of a new LSO HI-REZ PET/CT Scanner IEEE NSS \& MIC Conf. Rec. 3130-34

Jakoby B W, Bercier Y, Watson C C, J, Bendriem, Townsend D W 2009 Performance characteristics of a new LSO PET/CT scanner with extended axial field-of-view and PSF reconstruction IEEE Trans. Nucl. Sci 56 633-40

Joseph P M 1982 An improved algorithm for reprojecting rays through pixel images IEEE Trans. Med. Imag. vol. 192-6

Moehrs S, Defrise M, Belcari N, Del Guerra A, Bartoli A, Fabbri S, Zanetti G 2008 Multi-ray-based system matrix generation for 3D PET recosntruction Phys. Med. Biol. 53 6925-45

Mumcuoglu E, Leahy R, Cherry S and Hoffman E 1996 Accurate geometric and physical response modelling for statistical image reconstruction in high resolution PET IEEE NSS \& MIC Conf. Rec. 3 1569-73

Mustafovic S, Thielemans K. 2004 Object dependency of resolution in reconstruction algorithms with interiteration filtering applied to PET data IEEE Trans. Med. Imaging. $234433-46$

Ortũ no J E, Guerra P, Rubio J L, Kontaxakis G, and Santos A 2006 3D OSEM-based iterative image reconstruction for high resolution PET using precalculated system matrix Nucl. Instr. Meth.Phys. Research. 5692 440-4

Panin V, Kehren F, Michel C, Casey M 2006 Fully 3-D PET reconstruction with system matrix derived from point source measurements IEEE Trans. Med. Imaging. 25 907-21

Panin, V.Y., Kehren, F., Casey, M.E 2007 Incorporation of axial system response in iterative reconstruction from axially compressed data of a cylindrical scanner using on-the-fly computing IEEE NSS \& MIC Conf. ReC pp. 2185-2189

Qi J, Leahy R, P. M. Cherry S, Chatziioannou A, and Farquhar T 1998 High- resolution 3-D Bayesian image reconstruction using the microPET small-animal scanner Phys. Med. Biol., vol. 43 1001-13

Rahmim A, Lenox M, Michel C, Reader A J and Sossi V 2003 Space-variant and anisotropic resolution modelling for list-mode EM reconstruction IEEE NSS \& MIC Conf. ReC. 5 3074-7

Rahmim A, Tang J, Lodge M A, Lashkari S, Ay M R, Bengel F M 2008a Resolution Modeled PET Image Reconstruction Incorporating Space-Variance of Positron Range:Rubidium-82 Cardiac PET Imaging IEEE NSS \& MIC Conf. ReC. $3644-50$

Rahmim A, Tang J, Lodge M A, Lashkari S, Ay M R, Lautamaki R, Tsui B M W, and Bengel F M, 2008b Analytic system matrix resolution modelling in PET: an application to Rb-82 cardiac imaging Phys. Med. Biol, vol. 53 5947-65

Rapisarda E, Bettinardi V. Thielemans $\mathrm{K}$ and Gilardi M C 2010 Image-based point spread function implementation in a fully 3D-OSEM reconstruction algorithm for PET Phys. Med. Biol. 55 4131-51

Reader A J and Zweit J 2001 "Developments in whole body molecular imaging of live subjects" Trends in Pharmacological Sciences 22 604-7

Reader A J, Ally S, Bakatselos F, Manavaki R, Walledge R J, Jeavons A P, Julyan P J, Zhao S, Hastings D L and Zweit J 2002 One-pass list-mode EM algorithm for high-resolution 3D PET image reconstruction into large arrays IEEE Trans. Nucl. Sci. 49 693-9

Reader A J, Julyan P J, Williams H, Hasting D L, and Zweit J 2003 EM Algorithm System Modelling by Image-Space Techniques for PET Reconstruction IEEE Trans. Nucl. Sci. 505 1392-97

Saleem A, Ranson M, Callies S, Lahn M, Prenant C, Brown G, Matthews J C, Dence C S, McMahon A and Price P 2009 Journal of Clinical Oncology, 2009 ASCO Annual Meeting Proceedings 2715 S 3578

Siddon R L 1985 Fast calculation of the exact radiological path for a three-dimensional CT array Med. Phys. 12 252-5

Snyder D L, Miller M I, Thomas L J, Jr., and Politte D G 1987 Noise and edge artifacts in maximum-likelihood reconstructions for emission tomography," IEEE Trans. Med. Imaging MI-6, 228-38

Sossi V, Buckley K R, Piccioni P, Rahmim A, Camborde M L, and Ruth T J 2003 Printed sources for positron emission tomography (PET) IEEE NSS \& MIC Conf. Rec., Portland, OR

Sureau F, Reader A, Comtat C, Leroy C, Ribeiro M, Buvat I 2008 Impact of image-space resolution modelling for studies with the high-resolution research tomograph J. Nucl. Med.49 1000-8

Tohme M, Qi J, 2009 Iterative image reconstruction for positron emission tomography based on a detector response function estimated from point source measurements Phys. Med. Biol. 54 3709-25

Tomic N, Thompson C, Casey M, 2005 Investigation of the block effect on spatial resolution in PET detectors IEEE Trans. Nucl. Sci..52 599-605

Tong S, Alessio AM, Kinahan PE Noise properties in PSF-based fully-3D PET image reconstruction: an experimental evaluation 2010 Phys. Med. Biol. 55 1453-73

Van Staden J, Du Raan H, Lötter M, Van Aswegen A, Herbst C 2007 Production of radioactive quality assurance phantoms using a standard inkjet printer Phys. Med. Biol. 52 329-337

Watson C C, Casey M E, Michel C, and Bendriem B 2004 Advances in scatter correction for 3D PET/CT IEEE NSS \& MIC

Conf. Rec, 3008-12.

Wiant D B, Gersh J A, Bennett M C, Bourland J. D 2009 PET image reconstruction using LOR-OSEM with a 3D spatially variant system matrix |IEEE NSS \& MIC Conf. Rec $3752-3758$ 